\title{
Differential gene expression in the evolution of sex pheromone communication in New Zealand's endemic leafroller moths of the genera Ctenopseustis and Planotortrix
}

\author{
Alessandro Grapputo ${ }^{1 *}$ D, Amali H. Thrimawithana2 ${ }^{2}$ Bernd Steinwender ${ }^{2,3}$ and Richard D. Newcomb $b^{2,3}$
}

\begin{abstract}
Background: Sex pheromone communication in moths has attracted the attention of evolutionary biologists due to the vast array of pheromone compounds used, addressing questions of how this diversity arose and how male reception has evolved in step with the female signal. Here we examine the role of changing gene expression in the evolution of mate recognition systems in leafroller moths, particularly focusing on genes involved in the biosynthetic pathways of sex pheromones in female pheromone glands and the peripheral reception repertoire in the antennae of males. From tissue-specific transcriptomes we mined and compared a database of genes expressed in the pheromone glands and antennae of males and females of four closely related species of leafroller moths endemic to New Zealand, Ctenopseutis herana and C. obliquana, and Planotortrix excessana and P. octo. The peculiarity of this group, compared to other Lepidoptera, is the use of (Z)-5-tetradecenyl acetate, (Z)-7-tetradecenyl acetate, and (Z)-8-tetradecenyl acetate as sex pheromone components.
\end{abstract}

Results: We identify orthologues of candidate genes from the pheromone biosynthesis pathway, degradation and transport, as well as genes of the periphery olfactory repertoire, including large families of binding proteins, receptors and odorant degrading enzymes. The production of distinct pheromone blends in the sibling species is associated with the differential expression of two desaturase genes, deast 5 and desat7, in the pheromone glands. In male antennae, three odorant receptors, OR74, OR76a and OR30 are over-expressed, but their expression could not be clearly associated with the detection of species-specific pheromones components. In addition these species contain duplications of all three pheromone binding proteins (PBPs) that are also differentially expressed among species.

Conclusions: While in females differences in the expression of desaturases may be sufficient to explain pheromone blend differences among these New Zealand leafroller species, in males differential expression of several genes, including pheromone binding proteins, may underpin differences in the response by males to changing pheromone components among the species.

Keywords: Tortricids, Sex pheromones, Gene expression, Transcriptomics, RNAseq, Desaturases, Odorant receptors, Pheromone binding proteins

\footnotetext{
* Correspondence: alessandro.grapputo@unipd.it

${ }^{1}$ Department of Biology, University of Padova, Padova, Italy

Full list of author information is available at the end of the article
}

(c) The Author(s). 2018 Open Access This article is distributed under the terms of the Creative Commons Attribution 4.0 International License (http://creativecommons.org/licenses/by/4.0/, which permits unrestricted use, distribution, and reproduction in any medium, provided you give appropriate credit to the original author(s) and the source, provide a link to the Creative Commons license, and indicate if changes were made. The Creative Commons Public Domain Dedication waiver (http://creativecommons.org/publicdomain/zero/1.0/) applies to the data made available in this article, unless otherwise stated. 


\section{Background}

Moths rely on a sophisticated olfactory system for their survival and reproduction. Mate finding typically involves long-range attraction of males by sex pheromones emitted by females. Moth sex pheromones often consist of species-specific blends of modified long-chain unsaturated fatty acids with terminal functional groups as such alcohols, aldehydes, or acetate esters. Sex pheromone communication systems are considered to be under strong purifying selection to maintain the link between the females' pheromone and the males' ability to recognise only conspecific blends $[1,2]$. This has created a conundrum of how new pheromone blends arise, with both gradual evolution and saltatory shifts being suggested to explain the resulting species diversity we see today [3].

One of the challenges in understanding sex pheromone evolution is determining how male preference can change in step with the female signal. Pheromone evolution might reflect selection on the receivers rather than on the emitters because of the differential investment of the two sexes [4]. Therefore, according to Phelan's asymmetrical tracking hypothesis, selection on the female pheromone signal will be weak and females should have relatively large among-female variation in the pheromone blend. The corresponding response in males will track the female signals, but again selection should be weak. Males should show a wide breadth in pheromone responses to locate potential partners and maximise their fitness, this in turn will reduce the selection pressure on females $[2,4]$. Still other hypotheses include variants of sexual selection with males choosing among variable blends produced by females that may act as proxies for beneficial traits or females striving to be different and detecting these differences themselves [5].

Notwithstanding these various hypotheses, the different phenotypes favoured by selection of male and female traits can be obtained through a number of potential molecular evolutionary mechanisms. With the genes involved in pheromone production in the female and recognition in the males becoming better understood (see below), the modes of molecular evolution can be explored. One mode might include structural mutation of important proteins, while a second might involve changes in gene expression, including differential gene expression in the two sexes. Evidence has been accumulating on the importance of gene expression differences in reproductive isolation and rapid divergence [6-9]. Certainly sex-biased expression is widespread among organisms and sex-biased genes show unusually rapid sequence evolution and are often labile in their pattern of expression [10]. Many examples of reproductive isolation involve gene expression differences in the olfactory system [11] causing shifts in sex pheromone preference in insect populations [12-14].
The composition of sex pheromones is likely under the control of a small number of genes [15] and recently substantial effort has been invested in identifying the genes involved in pheromone biosynthesis [2]. Moth sex pheromones are generally synthesized de novo in specialized pheromone glands, modified membranes between the 8 th and 9th abdominal segments, by a battery of enzymes similar to those involved in fatty acid biosynthesis and other gland-specific activities [16-18]. Enzymes common to both pathways are acetyl-CoA carboxylase, fatty acid synthase, desaturases and specific chain-shortening enzymes. Gland-specific enzymes include reductases that reduce fatty acids to alcohols and acetyltransferases that acetylate fatty alcohols to generate acetate esters [16]. Most research has focused on the identification and characterization of desaturases [19-29], because the position of double bonds within the acyl chain underpins much of sex pheromone diversity. Most evidence has been found for differential gene expression between species (e.g. [27]), however some structural differences have also been described [29]. More recently fatty acid reductases (FARs) have also been targeted [30-34] with structural variants identified that differ in their ability to use various unsaturated substrates [33].

Pheromone reception in moths takes place within specialized hairs on the antennae, with sensilla each containing one to three olfactory sensory neurons (OSNs). Odorants penetrate the sensillum through pores binding to odorant binding proteins then receptors on OSN dendrites before finally being degraded to reset the system [35]. Four major families of membrane proteins are thought to be involved in odour reception in moths including odorant receptors (ORs), ionotropic receptors (IRs), and sensory neuron membrane proteins (SNMPs). Single amino acid changes responsible for specificity differences between species have been identified in pheromone sensing ORs [36]. Fewer differences have been found in gene families involved in transporting odorants to the receptors (e.g. odorant binding proteins and chemosensory proteins) or enzymes involved in resetting the system through hydrolysing odorants (e.g. carboxylesterases and glutathione-S-transferases).

Torticid leafroller moths of the sibling genera Ctenopseustis and Planotortrix are endemic to New Zealand and together with the Australian lightbrown apple moth, Epiphyas postvittana, form a complex of economically significant pests of fruit crops [37]. Both genera are widespread on the two islands and contain several closely-related species that are clearly differentiated by their sex pheromone composition [38]. The peculiarity of this group, compared to other members of the Tortricidae is the use of (Z)-5-tetradecenyl acetate (Z514:OAc), (Z)-7-tetradecenyl acetate (Z7-14:OAc), and (Z)-8-tetradecenyl acetate (Z8-14:OAc) as major sex 
pheromone components. Females of the sibling species C. herana and C. obliquana produce Z5-14:OAc and a blend of Z8-14:OAc and Z5-14:OAc in a 80:20 ratio, respectively [39]. Similarly, the sibling species $P$. octo and $P$. excessana produce a blend of $\mathrm{Z} 8-14$ :OAc and 14:OAc (98:2) and a blend of Z5-14:OAc and Z714:OAc (60:40), respectively [40, 41]. These female sex pheromone differences are mirrored by male speciesspecific responses. For example, males of $C$. obliquana are attracted by a blend of 90:10 of Z8-14:OAc and Z514:OAc, whereas $C$. herana males are attracted by Z514:OAc alone [42, 43]. The characterization of the molecular components involved in odorant reception, as well as the biosynthetic pathway of pheromone production in these moths is well underway. Recent research has focused on the isolation and characterization of desaturases showing that the production of these distinctive sex pheromones in closely related species involves the differential regulation of the desaturase gene desat $5[27,44]$. On the pheromone reception side two orthologous receptors, OR7 and OR30 display biased expression in male antennae compared to females in all four species. The receptor OR7 in particular shows signatures of positive selection and responds to Z814-OAc suggesting it acts as pheromone receptors in both genera $[45,46]$.

Here we use a combination of next generation transcriptome sequencing (RNAseq), bioinformatics and phylogenetic analyses to compare genes expressed in the antennae of both sexes and pheromone glands of $C$. obliquana, $C$. herana, $P$. octo and $P$. excessana. We identify orthologues of candidate genes of the pheromone biosynthesis pathway, as well as genes of the periphery olfactory repertoire, including large families of carrier proteins, receptors and odorant degrading enzymes. We use these data to compare modes of molecular evolution (sequence vs expression) in this mate recognition system.

\section{Methods}

\section{Sampling and RNA sequencing}

Four endemic species of New Zealand leafroller moths (Ctenopseustis herena, C. obliquana, Planotortrix excessana and $P$. octo) were obtained from the insect rearing facility at Plant \& Food Research, Mount Albert Research Centre, Auckland, New Zealand. The history of the laboratory strains has been reported previously [47]. A fifth species, the light brown apple moths (Epiphyas postvittana), for which mined curated genes and gene models from a genome assembly were available $[48,49]$, was used as an outgroup in the evolutionary analyses.

Antennae were dissected from 100 2-3-day-old male and virgin female adult moths of each species. Pheromone glands were dissected from the 100 virgin adult females of each species. RNA was extracted from each pool of tissues using $800 \mu \mathrm{l}$ of Trizol (Invitrogen, Carlsbad, CA, USA) following the manufacturer's protocol. After DNase treatment (DNaseI amplification grade, Invitrogen) cDNA was synthetized using the iScript cDNA Synthesis Kit (Bio-Rad, Hercules, CA, USA) from $1 \mu \mathrm{g}$ of total RNA, incubated at $25^{\circ} \mathrm{C}$ for $5 \mathrm{~min}, 42^{\circ} \mathrm{C}$ for $30 \mathrm{~min}$ and $85^{\circ} \mathrm{C}$ for $5 \mathrm{~min}$.

Pair-end RNAseq libraries were constructed from each male and female antennae and pheromone gland pools using Illumina's standard protocols and sequenced at Macrogen (Seoul, South Korea). Raw sequence data are available in the NCBI-SRA archive under the bioproject numbers: PRJNA236627, PRJNA236626, PRJNA243920, PRJNA243922 and PRJNA236624 respectively for C. herana, C. obliquana, P. excessana, P. octo and E. postivttana. Quality of the raw reads pairs for each library was assessed using FastQC (version 0.11.2) [50]. Prior to assembly mitochondrial contamination was removed by mapping the reads to a set of reference mitochondrial genomes of Tortricidae downloaded from NCBI, as well as the mitochondrial genomes of $C$. obliquana and $P$. octo using bowtie2 (version 2.2.5) [51], where reads mapping to the references were discarded. Thereafter the reads were screened for adapters and trimmed to a quality score threshold of 20 using fastq-mcf from the eautils package (version 1.1.2-806) [52]. An in-house perl script was then used to trim 15 bases of their $5^{\prime}$ end and to discard reads containing Ns and mononucleotides. Finally, possible contaminants from bacteria and fungi were screened out using Kraken with the Refseq fungal and bacterial databases (version 0.10.5-beta) [53].

\section{Assembly and annotation}

Assemblies were conducted independently for each species by combining sexes and tissues for each species. We conducted several assemblies for each species using SOAPdenovo-trans (version 1.04) [54] with kmer series of 31-75 with increments of four bases and Trinity (version 2.0.6) [55]. For C. obliquana and P. octo for which a partial draft genome sequence was available (unpublished data) we carried out both a de novo and a genome-guided assembly with Trinity. These two assemblies were combined using PASA [56]. Final optimized and cleansed transcriptome assemblies were obtained combining all SOAP and PASA assemblies with EvidentialGene, tr2aacds perl script [57]. The CDS sequences of the "okayset" of EvidentialGene were used for the subsequent analyses and from now on are referred to as genes. Assemblies are available in the NCBI-TSA archive under the same bioproject numbers indicated above.

Orthologous genes among the five species were identified using ProteinOrtho (version 5.11) [58] with default parameters. 
Gene annotation was obtained by sequence similarity searches using NCBI blastx [59] against the nonredundant (nr) peptide database (including all nonredundant GenBank CDS translations + PDB + SwissProt + PIR+ PRF) with E-value $1 \mathrm{e}^{-4}$ within the Blast2Go package (version 3.1). A maximum of 10 hits were considered for each query and the E-value of $1 \mathrm{e}^{-4}$ was used to maximise the number of hits. Functional categories were assigned by mapping Gene Ontology (GO) terms using Blast2GO [60] with default parameters (E-values $1 \mathrm{e}^{-6}$, annotation cut-offs $>55$, GO weight $>5$ ). InterProScan annotation was also conducted via Blast2GO. Information obtained for domains were included to improve global annotations.

\section{Evolutionary analysis}

Sequences with blastx hits (cut off E-value $10^{-4}$ ) to gene families of odorant binding proteins, ionotropic receptors, sensory neuron membrane proteins, chemosensory proteins, carboxylesterases, pheromone binding activating neuropeptides, elongation of very long chain fatty acids proteins (ELOVLs), fatty acyl reductase (FAR), acetyltransferases, fatty acid synthases (FAS), fatty acid transport proteins (FATP), fatty acid amide hydrolases (FAAH) and acetyl-coa carboxylases (ACC) were extracted from the data sets and codon based aligned with Clustal W [61] in Geneious ver. 8.1 (Biomatters). The presence of multiple contigs in orthologous groups identify by ProteinOrtho suggested the existence of duplicated genes. Sequences were therefore manually curated and short sequences and putative alleles excluded. A phylogenetic tree for each gene family was obtained with FastTree ver.2.1 [62] from the protein sequences using the JTT model of evolution. The Shimodaira-Hasegawa test was used to compute the local support values at the nodes.

Phylogenetic clusters were compared to the previous orthologous groups obtained with ProteinOrtho to confirm duplicated genes and each group, whenever possible, tested for evidence of positive selection using the Codeml program in the PAML package [63]. Nested models M0-M3, M1-M2 and M7-M8 were compared using a likelihood ratio test (LRT) as twice the log-likelihood difference between the nested models. Significance was assessed by a $X^{2}$ test with the difference in the number of parameters of the models as the degrees of freedom.

\section{Differential expression}

The barcoded sequence library of each tissue (female antennae, male antennae and pheromone glands) of New Zealand endemic leafroller moths were individually aligned to the reference genes of their own species using Bowtie (version 2.2.5) [64] and counted using HTseq ver. 0.6.1p2 [65]. Each barcoded library was also mapped back to the curated genes and counted with HTseq. We used the Bioconductor package, DESeq2 (version 1.12.2) [66] to perform the differential expression analysis. We did not have RNAseq data available for the whole body of any of the species, therefore we assessed the biased expression relative to the other tissue (antennae or pheromone glands). Since we did not have within species replicates we applied the variance-stabilizing transformation to the raw read counts and then calculated the log fold change between pairs of samples within species using a threshold of 2 to consider a gene as biased expressed. Enrichment of GO terms in the biased expressed genes was assessed using Piano [67] an $R$ package that collects several Gene Set Analyses (GSA) methods and allows consensus scoring of the results of the multiple GSA runs. These methods in Piano make use of the full data set as they do not require of a priori cut-off of gene significance. We used six methods for calculating the gene set statistics, mean, median, sum, maxmean, GSEA and PAGE using the $\log$ fold change as input.

\section{Results}

Transcriptome assemblies and orthologous genes

Illumina RNAseq produced an average of about 67.4 million reads $(\mathrm{SE} \pm 3.6$ million) per species and tissue (Table 1). After trimming and mitochondrial and contaminant removal, we were left with an average of 53.6 million ( $\mathrm{SE} \pm 3.5$ million) reads per species and tissue, corresponding to an average of $79 \%(\mathrm{SE} \pm 2.76 \%)$ of the initial raw reads (Additional file 1: Table S1).

The number of contigs (or genes according to Trinity) produced by different assembly algorithms and kmer sizes ranged from 10,638 (P. octo, SoapTrans k75) to 173,448 (C. herana, SoapTrans k31). The assembly statistics for the different algorithms and kmer sizes are reported in supplementary material (Additional file 2: Table S2). Scaffolds and genes obtained with SoapTrans and Trinity (both denovo and guided) were combined with the EvidentialGene tr2aacds.pl pipeline script into a set of optimized and cleansed transcriptome assemblies. These assembles consisted of 24,989,20,840,23,304,20,883 and 23,591 coding sequences (CDS) for C. herana, C. obliquana, $P$. excessana and $P$. octo, respectively (Additional file 3: Table S3). These numbers are comparable with those found with the Evidentialgene method in Nasonia vitripennis, but higher than those found in Bombyx mori, see [57] for a comparative table. From 53\% (P. octo: 11,055$)$ to $58 \%$ (P. excessana: 13,566) of these CDS were considered full length by EvidentialGene.

For the CDS, 15,896 (63.61\%), 14,318 (68.70\%), 14,610 $(62.69 \%)$ and $14,133(67.68 \%)$ showed similarity (Blastx, E-value $1 \mathrm{e}^{-4}$ ) to protein sequences in the NCBI non- 
Table 1 Summary statistics of blastx hits and GO term annotations

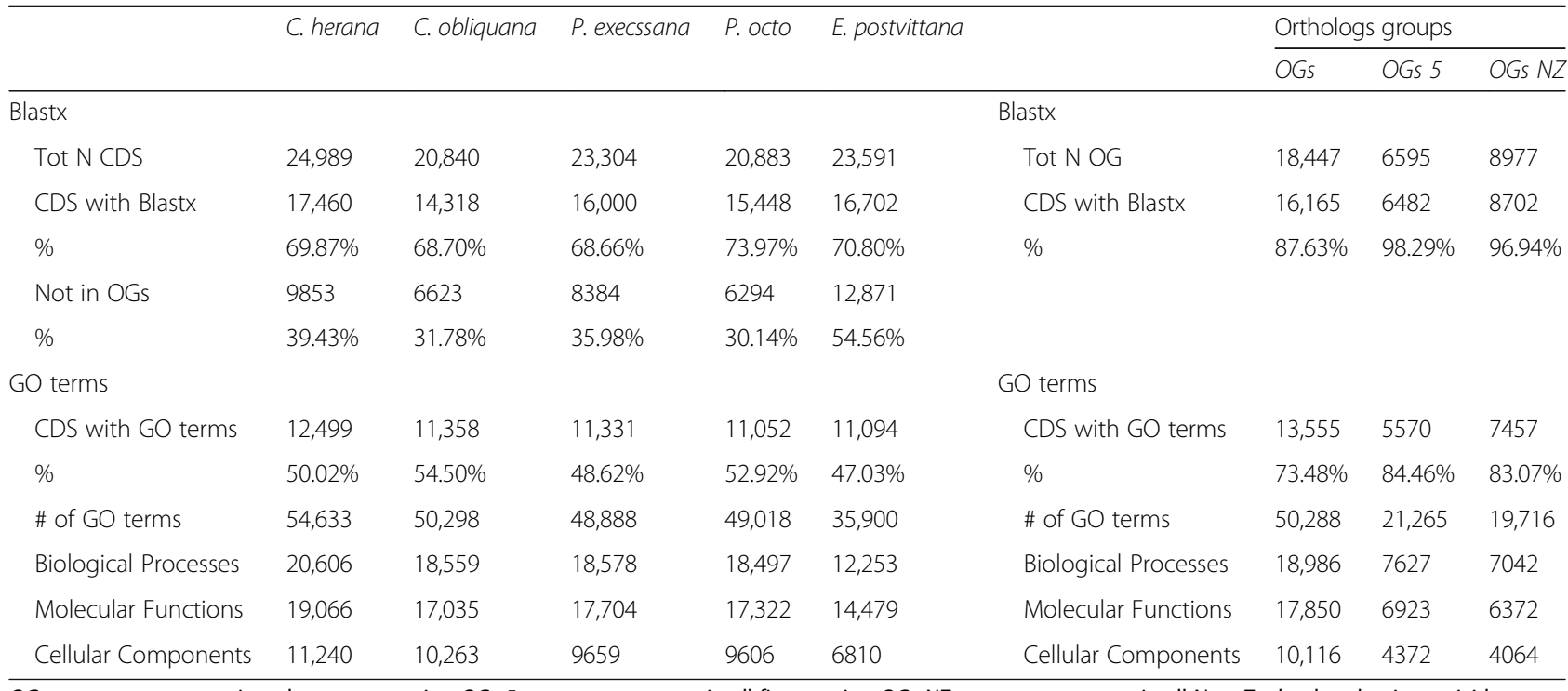

OGs sequences present in at least two species, OGs 5 sequences present in all five species, OGs NZ sequences present in all New Zealand endemic tortricids

redundant protein database. Despite the relative high Evalue cut-off used about $30 \%$ of the contigs of each species remained without any hits. The number of genes assigned to functional groups (GO terms) for C. herana, C. obliquana, P. excessana and P. octo were 12,499, $11,358,11,331$ and 11,052, respectively (Table 1 ). For all species, the most abundant GO categories within Biological Process were the "macromolecule metabolic process" and the "cellular macromolecule metabolic process" both represented by $13-16 \%$ of the genes. The most abundant GO categories with Molecular function were "nuclear acid binding" and "cation binding" representing about $11 \%$ of the genes in both cases. Finally, within Cellular Components the most abundant GO categories were "intracellular membrane-bounded organelle" and "cytoplasm" represented by $9-10 \%$ and $7-8 \%$ of the genes, respectively. A complete list of blastx descriptions, best hits and GO annotations for each species is given in Additional file 4: Table S4 and the GO distribution in the three categories of each species is shown in the Additional file 5: Figure S1.

Comparing the translated coding sequences among the moth species, including E. postvittana, we identified 18,447 orthologous groups (Additional file 6: Table S5), of which 5644 showed a simple one to one relationship in all species, 184 had multiple orthologs in at least two of the species and in 11,852 cases orthologs were missing in at least one of the species. A significant blast hit (Blastx, E-value $1 \mathrm{e}^{-4}$ ) was obtained for 16,165 (87.63\%) of the groups. Out of the total number of orthologous groups, 6595 contained at least one sequence from each of the five moth species and $98.29 \%$ (6482) of these had a significant blast hit (Table 1). The proportion of coding sequences that were not assigned to any orthologous group ranged from 30 to $40 \%$ in the New Zealand leafroller moths, reaching $54 \%$ for $E$. postivittana.

\section{Biased gene expression}

The Evidentialgene CDS sequence set for each species was used as a reference transcriptome for quantifying the expression levels of genes in female antennae, male antennae and pheromone glands. We mapped reads to CDS instead of transcripts to increase the accuracy of read assignment to putative genes and kept distinct putative CDSs found in long transcript UTRs. The gene expression values for each gene and species are given in Additional file 5: Table S4.

The number of expressed genes in the different tissues varied among species. In $C$. herana and $P$. octo the greatest number of expressed genes was found in the pheromone glands, whereas in C. obliquana and P. excessana the greatest number of expressed genes was in male antennae (Table 2). The difference in number of expressed genes among tissue and species was not related to sequence depth.

In the comparison of gene expression between antennae of the two sexes we also included E. postvittana. Few genes were differentially expressed between male and female antennae, with the exception of $P$. excessana where 227 displayed greater expression in female antennae. In all species, male antennae biased genes were mostly olfactory receptors and pheromone binding proteins. Two olfactory receptors, OR7 and OR30, were the most male-biased in their expression in all five species (Additional file 7: Table S6). Two pheromone binding protein (PBP1 and 3) were also male biased in all species except in P. excessana. Finally, two lipases (OG3363 in the New Zealand moths and OG362 in E. postvittana) 


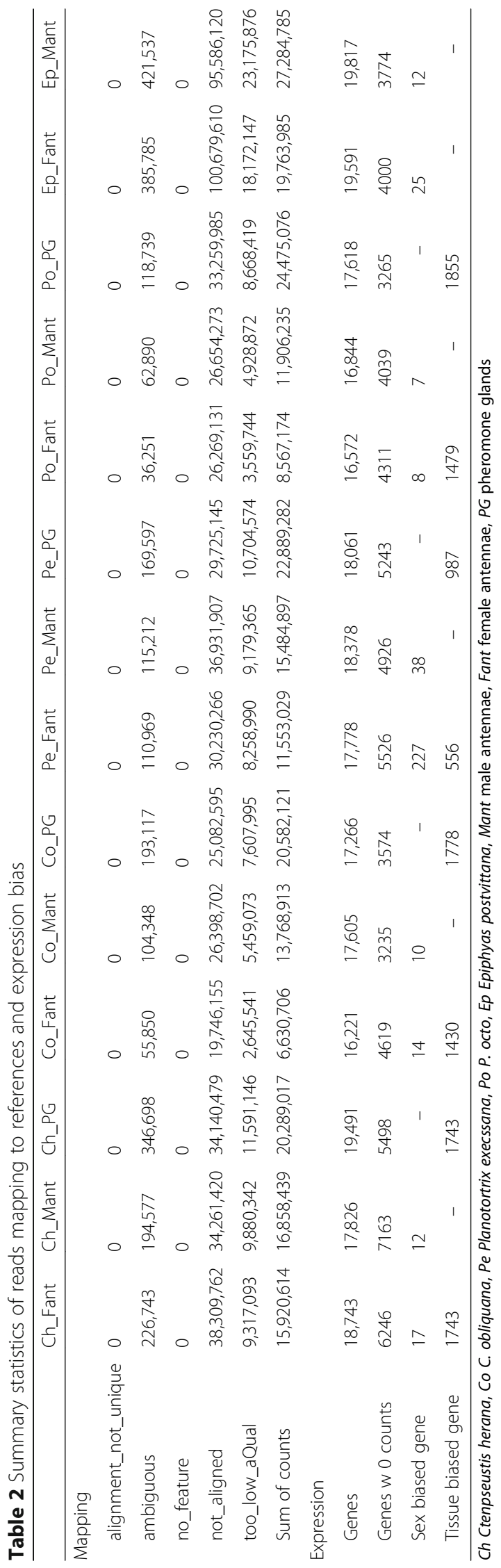


display greater expression in male antennae making them candidate sex pheromones degrading enzymes. The olfactory receptor OR4 was female biased in the antennae of all species. Another olfactory receptor (OR21) was biased in female antennae in all species except $E$. postvittana. Three female biased genes were shared between the Ctenopseustis species; a cytochrome p450 (OG4842), one hypothetical protein (OG11897) and an unknown transcript (OG15104). Planotortrix females also shared three antennal-biased genes; a cytochrome p450 (OG8571) a predicted protein (OG6394) and an unknown transcript (OG13098) (Additional file 7: Table S6). GSA analysis did not show common enriched GO terms for either male or female antennae among all species (Additional file 8: Table S7, Additional file 9: Figure S2).

No transcriptome is available for E. postvittana pheromone glands, therefore the comparison among pheromone gland transcriptomes only considered the four New Zealand endemic leafroller moths. Here, biased expression is relative to the other tissue (antennae or pheromone glands) as no RNA-seq data was available for the whole body. The number of differentially expressed genes comparing female antennae and pheromone glands ranged from 1543 in P. excessana to 3334 in $P$. octo, with about half biased in their expression in either antennae or pheromone glands. Biased expression in female antennae was observed within 2307 orthologous groups of which 402 had a gene in each species (Fig. 1a). The annotated genes with the highest expression bias included mainly odorant binding proteins
(OBPs), pheromone binding proteins (PBPs) and general odorant binding proteins (GOBPs), which are described in the mined gene section below. Among the top 20 most biased genes common in female antennae we also found an unknown protein (OG2944) with a twelve-fold change in expression, one hypothetical protein (OG11897) similar to Danaus plexippus EHJ71609 and a glutamate receptor kainate 2 (OG451). Expression values in female antennae for all genes are reported in Additional file 5: Table S4. In all species, genes displaying female biased expression in antennae were over represented in gene ontology (GO) terms mostly related to sensory perception (Additional file 10: Table S8, Additional file 11: Figure S3). In the Biological Process category, among the most enriched GOs were $G$ protein-coupled receptor signalling pathway (GO:0007187), detection of chemical stimulus involved in sensory perception of smell (GO:0050911), sensory perception of smell (GO:0007608) and ionotropic glutamate receptor signalling pathway (GO: 0035235). In the Molecular Function category, over represented GO terms were odorant binding (GO: 0005549), olfactory receptor activity (GO: 0004984) and ionotropic glutamate receptor activity (GO: 0004970). The most significant over represented GO terms in the Cellular Component included membrane (GO:0016020), intraciliary transport particle B (GO: 0030992) and other GO terms related to membrane and cilium.

We found 2729 orthology groups with biased expression in pheromone glands, of which 278 had a gene in each species (Fig. 1b). Genes with the most biased expression in pheromone glands included a vitellogenin
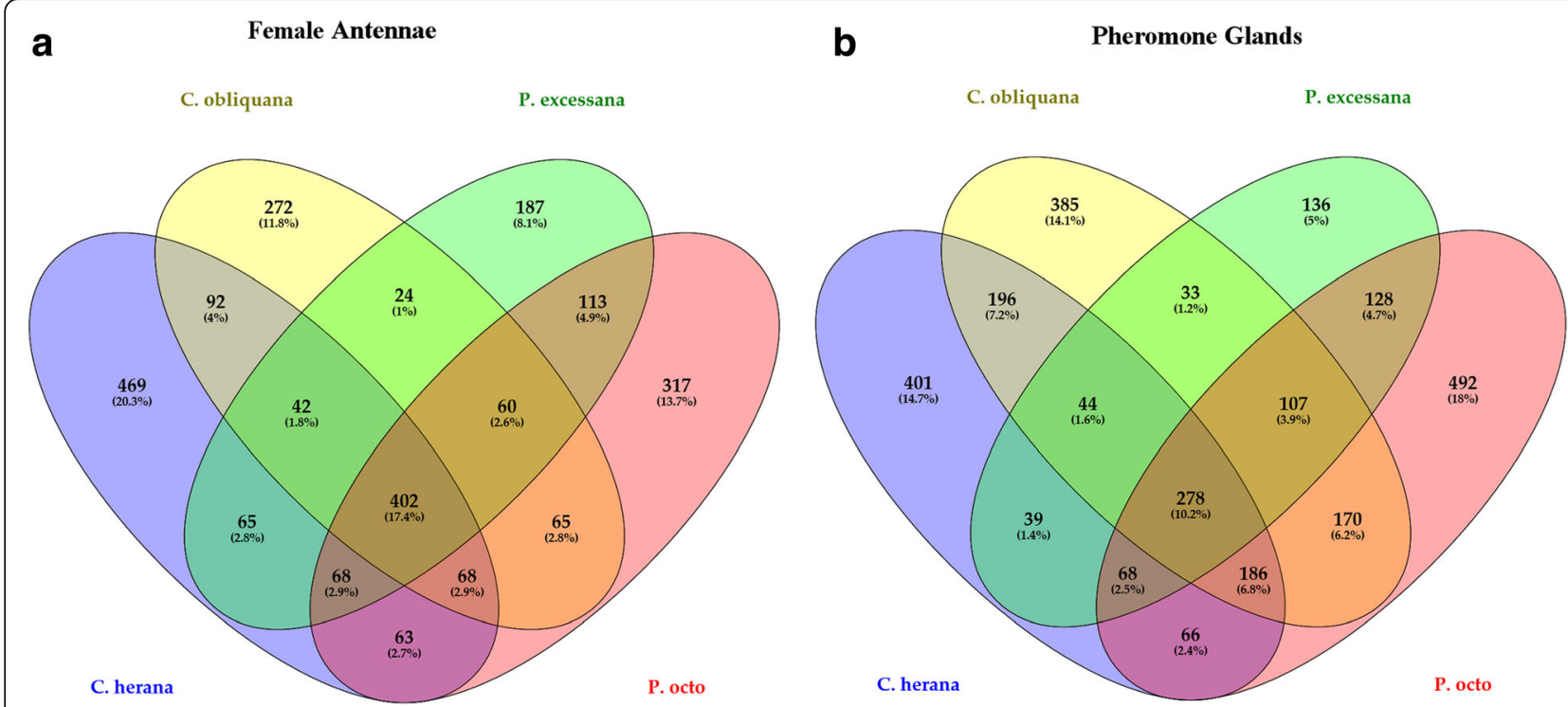

Fig. 1 a Venn diagram showing the number of orthologous groups shared between female antennae of the four New Zealand leafroller moths, Ctenopseustis herana, C. obliquana, Planotortrix excessana and P. octo. $\mathbf{b}$ Venn diagram showing the number of orthologous groups shared between pheromone glands of the four New Zealand leafroller moths 
(OG4241), a juvenile hormone binding protein (OG4718) and a takeout protein (OG3767), although none of them was among the most biased genes in all four species (Additional file 5: Table S4). Among the genes with biased expression in pheromone glands we also found three desaturases (OG14247, 13,411 and $10,612)$ corresponding to the desaturases desat2, desat4 and desat5 described in [27], two alcohol dehydrogenases (OG11355 and 13,787), three cytochrome P450s and two takeout-like protein 2, a CSP (CSP6a-OG3423) and an odorant binding protein (OBP17). A more detailed description of biased genes with putative function in pheromone biosynthesis, degradation and transport is reported in the mined gene section below. In all species biased expressed genes in pheromone glands showed over represented GO terms mostly related to fatty acid synthase (Additional file 10: Table S8, Additional file 11: Figure S3). In the Biological Process category, among the most over represented GO term in pheromone glands was oxidation-reduction process (GO:0055114) in all four species. Oxidoreductase activity (GO:0016491) was among the most over represented GO terms in Molecular Function, while fatty acid synthase complex (GO:0005835) was in Cellular Component. Ctenopteustis herana, and to some extent $P$. excessana, showed enrichment in the Molecular Function for acyl-carrier proteins (GO:0004313, GO:0004314, GO:0004315, GO:0004316, GO:0004317, GO:0004319, GO:0004320, GO:0016295, GO:0016296).

\section{Manual curation of gene families and evolutionary analyses}

We focused on the genes of the pheromone biosynthesis pathway and peripheral olfactory repertoire and proceeded to mine all genes with blast hits for the gene families described in the following paragraphs. Diagrams of the pheromone biosynthesis pathway and pheromone reception system with the putative genes involved are presented in Figs. 2 and 3, respectively. The list of genes and their expression values in the different tissues are

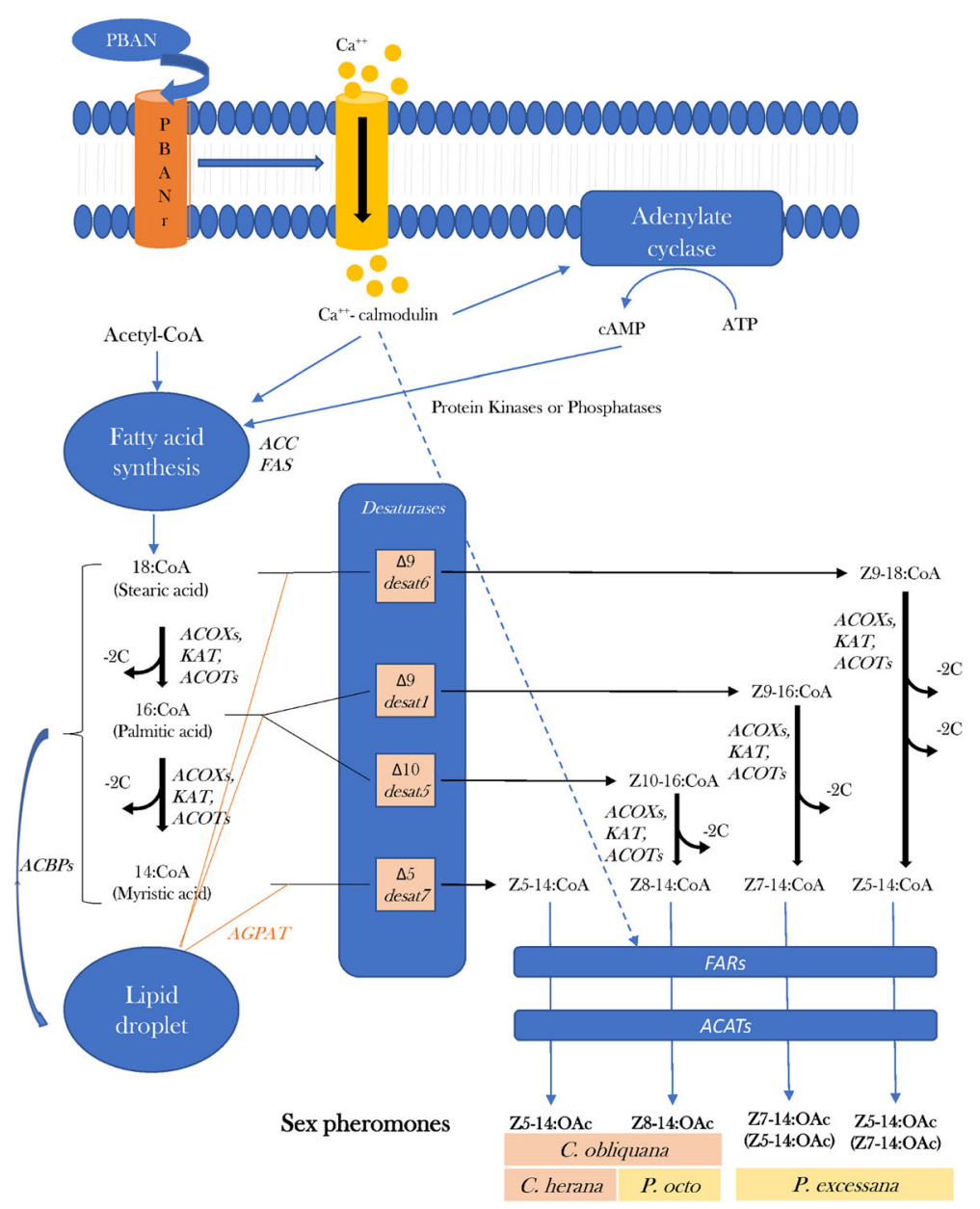

Fig. 2 Schematic view of the pheromone biosynthesis pathway in the four New Zealand leafroller moths, Ctenopseustis herana, C. obliquana, Planotortrix excessana and $P$. octo and the putative genes involved 


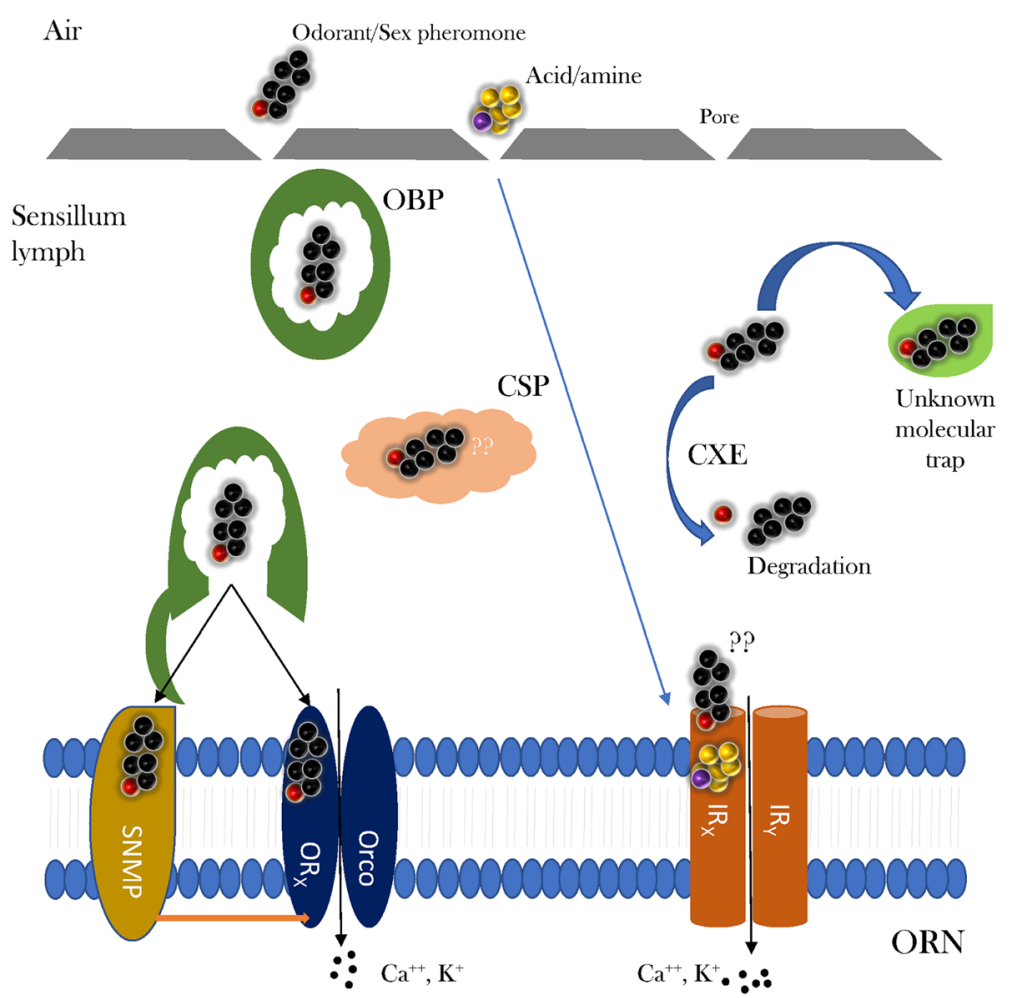

Fig. 3 Schematic view of the mechanisms involved in odorant binding, release and inactivation in moth antennae highlighting the gene families mined in this study

reported in Additional file 12: Table S9 and the analyses of positive selection in Additional file 13: Table S10 with a summary in Table 3. The nucleotide sequences of the mined genes are included in the Additional file 14.

Pheromone biosynthesis activating neuropeptide (PBAN) and pheromone biosynthesis activating neuropeptide receptor (PBANr)

Five sequences, one for each species of leafroller moth, had a good match to PBAN. The sequences had on average an amino acid identity of $48 \%$ with $B$. mori PBAN. A transcript of the PBAN receptor (PBANr) was found in all species except $E$. postivittana, for which we did not have a pheromone gland transcriptome, but we recovered the gene sequence from the genome (unpublished data). In the New Zealand leafroller moths the PBAN receptor was over expressed in the pheromone gland compared to antennae (Additional file 12: Table S9). No evidence for positive selection was detected for either gene using Codeml.

\section{Fatty acid synthase (FAS) and acetyl-CoA carboxylase (ACC)}

The first step of sex pheromone production is the synthesis of saturated fatty acids by acetyl coenzyme (CoA) carboxylase and fatty acids synthase (FAS). We found only a short fragment of about 900 bp similar to FAS corresponding to the sites between positions 4800 and 5700 of the D. melanogaster FAS CDS which totals about $7 \mathrm{~kb}$. This group of orthologous genes showed consistently higher counts $(\operatorname{LogFC}>2)$ in the pheromone gland compared with female antennae across all four endemic New Zealand species (Additional file 12: Table S9). The full CDS of about $7 \mathrm{~kb}$ of the acetyl CoA carboxylases (ACC-OG10860) was found in the endemic New Zealand moths, whereas only two fragments (for a total of about $1.1 \mathrm{~kb}$ ) were found in the antennal transcriptomes of E. postvittana, although the full CDS was obtained from the genome (unpublished data). ACC is a multi-domain enzyme that catalyses the ATP-dependent carboxylation of acetyl-CoA to malonyl-CoA, providing the substrate for the biosynthesis of fatty acids catalysing the synthesis of palmitic acid (C16:0) [68]. Malonyl-CoA is also the substrate for distinct elongases in the pathway of very long-chain fatty acyl-CoA synthesis [69]. ACC was more highly expressed in the pheromone gland compared to antennae in all New Zealand species except $P$. excessana in which the Transcripts Per Kilobase Million (TPM) in the pheromone glands was about ten times lower in expression than the other species (Fig. 4). This result is consistent with the previous finding that suggested that in P. excessana and Epiphyas postvittana 
Table 3 Summary of gene families involved either in pheromone biosynthesis or reception for presence of positive selection and expression bias between antennae in the two sexes and between pheromone gland and antennae in females

\begin{tabular}{|c|c|c|c|c|}
\hline & & Selection & Expression & \\
\hline & Gene Famyly & PAML & Sex - Antennae & $\begin{array}{l}\text { Female Gland/ } \\
\text { Antennae }\end{array}$ \\
\hline \multirow{13}{*}{$\begin{array}{l}\text { Pheromone } \\
\text { pathway }\end{array}$} & PBAN & - & & - \\
\hline & PBANr & - & & $>2$ \\
\hline & FAS & - & & $>2$ \\
\hline & ACC & - & & $>2$ \\
\hline & ACOX & - & & $>2$ \\
\hline & Desaturase & - & & $>2,>8$ \\
\hline & FAR & - & & $\pm>2,>8$ \\
\hline & ACAT & - & & $>2$ \\
\hline & ACBP & - & & $\pm>2$ \\
\hline & FATP & - & & - \\
\hline & FAAH & - & & $>2$ \\
\hline & FAT & - & & $\pm>2$ \\
\hline & ELOVL & - & & $\pm>2$ \\
\hline \multirow{6}{*}{$\begin{array}{l}\text { Pheromone } \\
\text { reception }\end{array}$} & OBP & + & $\pm>2$ & \\
\hline & CSP & - & - & \\
\hline & SNMP & - & - & \\
\hline & $O R^{*}$ & + & $\pm>2$ & \\
\hline & IR & - & - & \\
\hline & CXE & + & - & \\
\hline
\end{tabular}

+ gene families containing genes under positive selection

$>$ \# indicate log fold change

\pm gene families containing genes with bias expression in both tissues

*Analysis from [46]

synthesis and desaturation of the pheromone occurs outside the pheromone gland [70]. No evidence for positive selection was found for any of these genes.

\section{Desaturases}

Fatty-acyl desaturases introduce double bonds at specific carbon positions along the backbone of pheromone precursors and therefore are considered a major player in the diversity of sex pheromones. The analysis of the transcriptomic and genomic data did not reveal new genes other than the seven desaturases already described by $[27,44,71]$, therefore we redirect the reader to those papers for the results. However, while these previous publications found that desat 5 was expressed in C. herana, they did not present its sequence, which is now reported here. The analysis of both transcriptomic and genomic data of Planotortrix species failed to find an orthologue of desat7 in this genus. Desat7 has been described as a divergent desaturase by [71], clustering away from other desaturases. In Fig. 5 we show the phylogenetic relationships of the seven Ctenopseustis and Planotortrix desaturates with desat7 clustering with several desaturases of other moths (e.g. Plutella xylostella and Amyelos transitiella) and aphids (e.g Acyrthosiphon pisum) in a group that is sister to $\Delta 11$ desatusares of non-lepidopteran insects. The expression of these desaturases in pheromone glands and female antennae are presented in Fig. 4.

\section{Acyl-CoA oxidases (ACOX), 3-oxoacyl-CoA thiolase (KAT) and Acyl-CoA thioesterases/hydrolases (ACOTs)}

Modification of pheromone precursors involve chain shortening by $\beta$ oxidation enzymes. This step has not been well characterized in insects but most likely is similar to vertebrates. $\beta$-oxidases act in concert with a 3 oxoacyl-CoA thiolase to chain shorten acyl-CoAs by removing an acetyl group [18]. We found 35 sequences with similarity to acyl-CoA oxidases (COAX1 and 3) from which 11 were excluded after further inspection because they were too short or, after correction, were identical to other transcripts. The phylogenetic relationships of the remaining 24 sequences are shown in Additional file 15: Figure S4. The tree shows two main clusters which correspond to $B$. mori ACOX1 and 3. Three clusters of ACOX1 ( $a, b$ and $c$ ) were found in the leafroller moths compared with four found in $B$. mori. Two copies of ACOX3 (a and b) were found both in the leafroller moths and B. mori, however in E. postvittana we failed to find a transcript for ACOX3a (OG11206), but recovered the full CDS from the genome. An orthologous group (KAT), with one full CDS sequence per species, had $78 \%$ similarity to B. mori 3oxoacyl-CoA thiolase (XP_004930405). No evidence of positive selection was observed for any of the oxidase and thiolase genes. Although the four species of $\mathrm{New}$ Zealand tortricids investigated display different routes of pheromone biosynthesis (Fig. 2), ACOX3a was the only $\beta$-oxidase with biased expression in the pheromone glands and no differences were observed among species (Additional file 12: Table S9). ACOTs (or acyl-CoA hydrolases) are a group of enzymes that catalyze the hydrolysis of short to long-chain acyl-CoAs and promote $\beta$-oxidation by regulating the levels of free coenzyme $A$ $(\mathrm{CoASH})$ for the thiolase reaction [72]. $\mathrm{CoASH}$ is also required for the final step of $\beta$-oxidation, the 3-ketoacylCoA thiolase reaction (see below). Two thioesterase groups were found in leafroller moths, one more similar to palmitoyl-protein thioesterase (ACOT2) and the other to acyl-protein thioesterase (ACOT1). In $H$. virescens palmitoyl-protein thioesterase is over-expressed in pheromone glands [26], however in all leafroller moths both thioesterases were equally expressed in antennae and pheromone glands (Additional file 12: Table S9). No evidence of positive selection has been observed for either of these genes. 


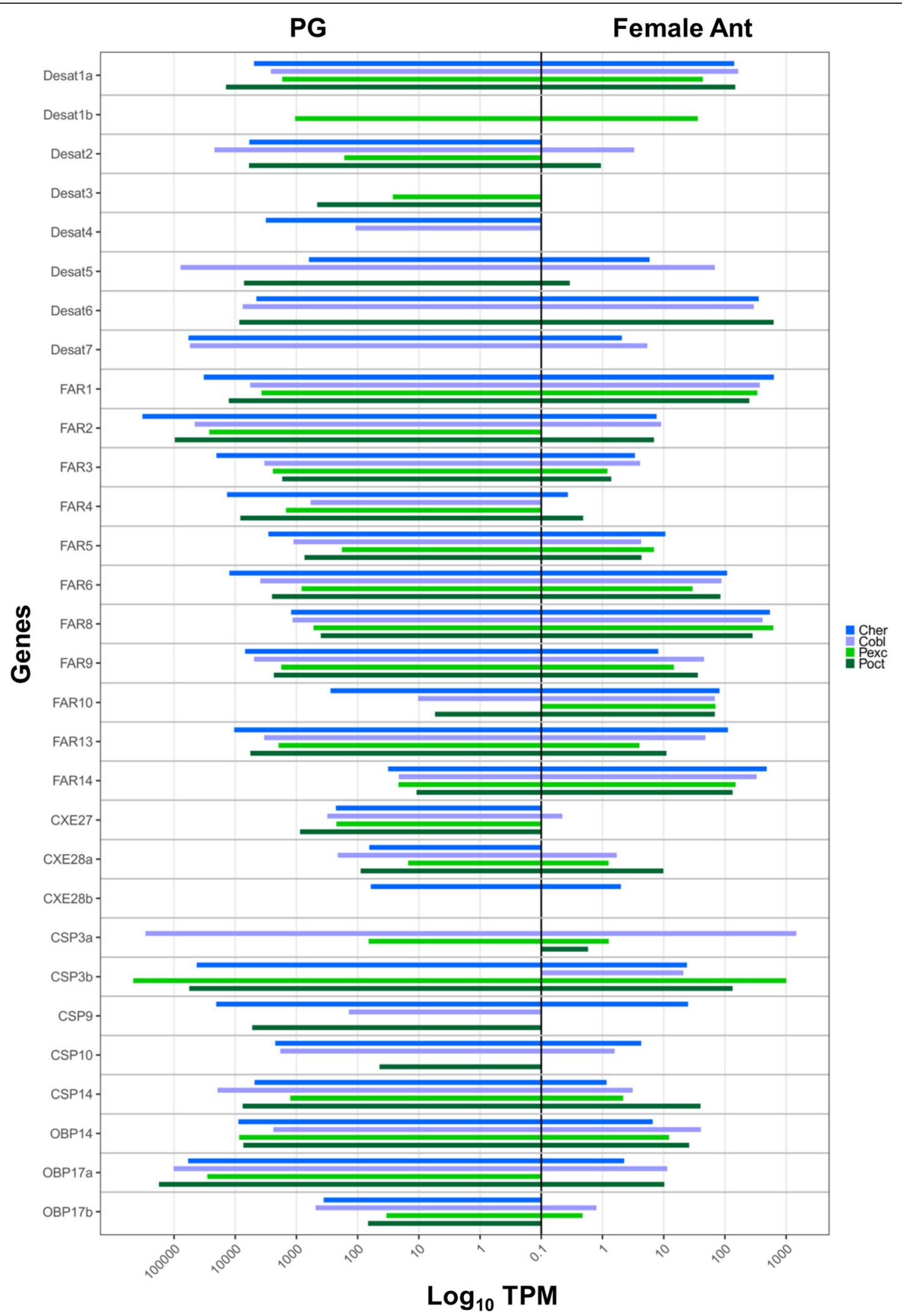

Fig. 4 Gene expression in Transcripts Per Kilobase Million (TPM) of desaturares, reductases and genes of the peripheral odorant reception repertoire with biased expression in the pheromone glands compared to antennae in the four New Zealand native leafroller moth species. Only genes showing a log fold change over a threshold of 2 in at least one species are shown 


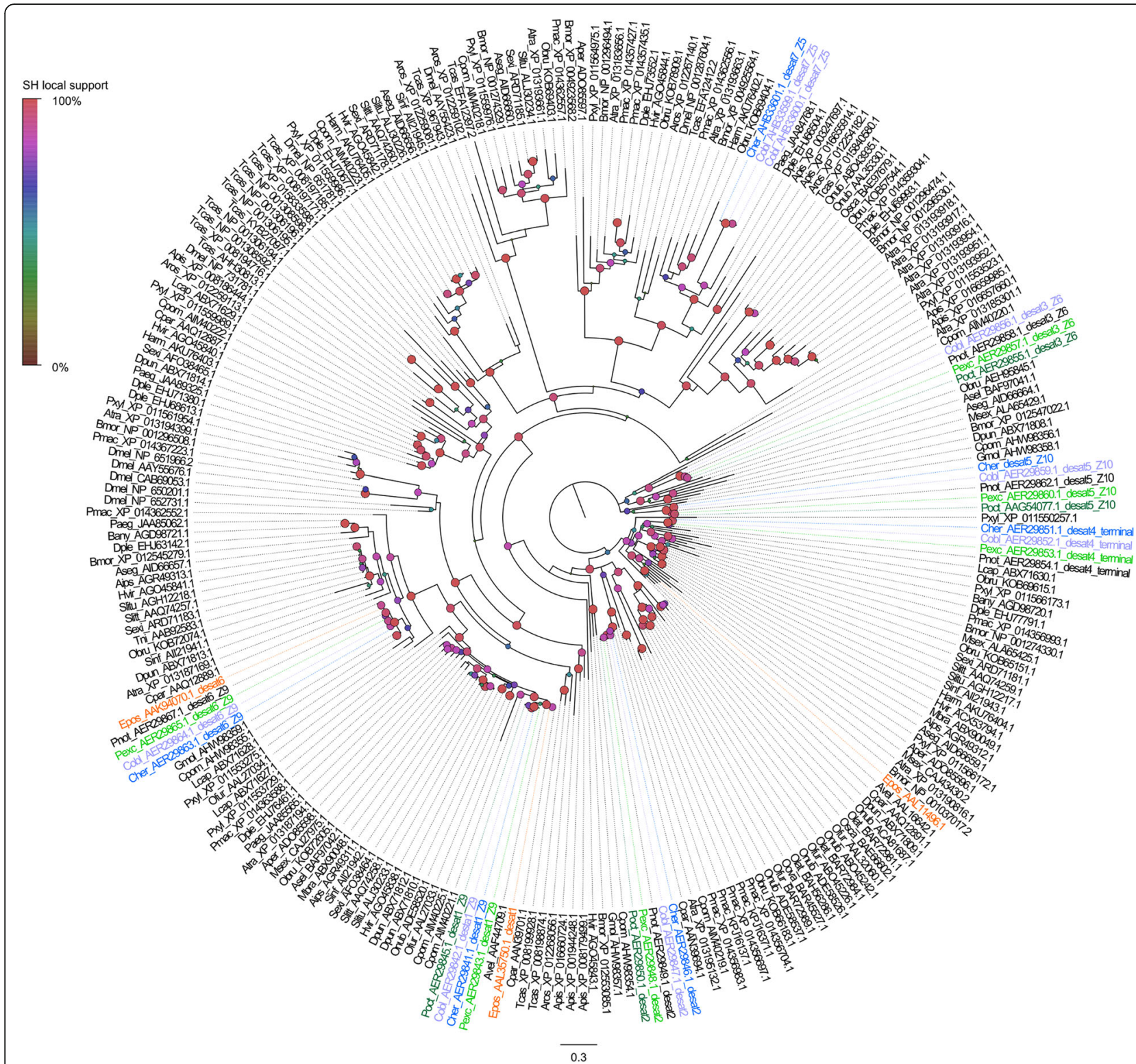

Fig. 5 Maximum likelihood tree showing the evolutionary relationships among desaturases retrieved from GenBank from available Lepidoptera, including the New Zealand leafroller moths Ctenopseustis herana (Cher, highlighted in blue), C. obliquana (Cobl, highlighted in light purple), Planotortrix excessana (Pexc, highlighted in light green) and P. octo (Poct, highlighted in dark green) and the horticultural pest Epiphyas postvittana (Epos, highlighted in orange) and Drosophila melanogaster (Dmel), Tribolium castaneum (Tcas) and Acyrthosiphon pisum (Apis). Circles at nodes indicate Shimodaira-Hasegawa local support, with colours and size of circles being proportional to the percentage of support (0-100\%)

\section{Fatty acid reductases (FAR)}

Once the specific double bond of the pheromone intermediate is produced, in tortricids the carbonyl carbon is typically modified to an acetate ester. This first requires the reduction of a fatty-acyl precursor to an alcohol by a fatty acid reductase [18]. One hundred and eight sequences had a blast hit with FARs. After manual curation 30 sequences were excluded from further analyses because they were too short or after correction, were identical to other transcripts. The phylogenetic relationship among the FARs is given in Fig. 6. The tree shows
15 clusters of orthologous sequences. For four clusters we failed to find an orthologue in E. postvittana. Three orthologous groups FAR2, 3 and 4 showed the greatest expression in the pheromone glands compared to the antennae in all four New Zealand endemic moth species, with $\log$ fold changes ranging from nine to 33 (Fig. 4, Additional file 12: Table S9). FAR2 was closely related to Ostrinia scapulalis OscaFAR XIII and B. mori pgFAR, which are pheromone gland specific in both species [30, 31] and the main determinant of the phenotypic variation in female pheromone between the 


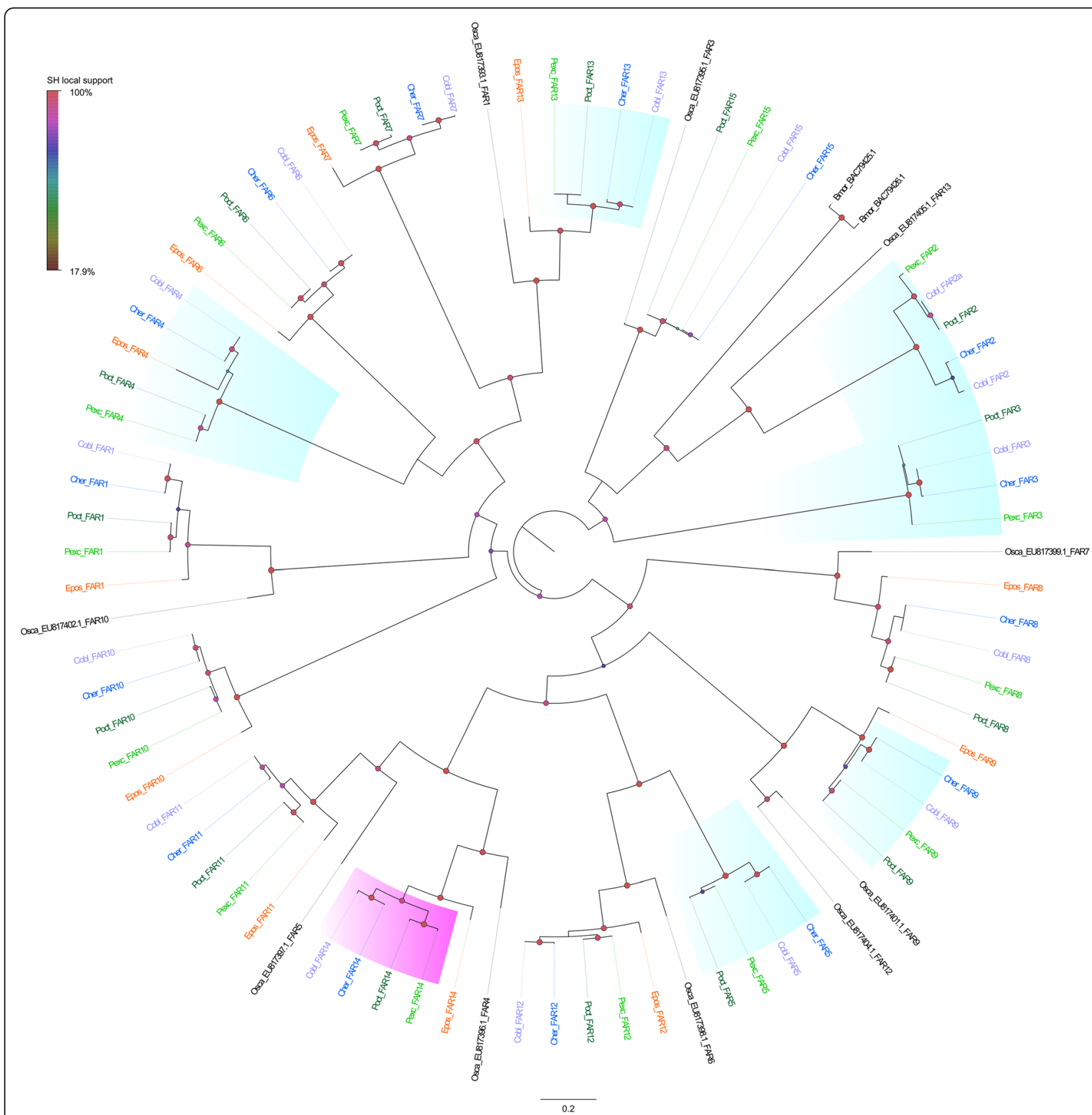

Fig. 6 Maximum likelihood tree showing the evolutionary relationships among fatty acid reductase (FAR) proteins mined from the transcriptomes of the New Zealand leafroller moths Ctenopseustis herana (Cher, highlighted in blue), C. obliquana (Cobl, highlighted in light purple), Planotortrix excessana (Pexc, highlighted in light green) and P. octo (Poct, highlighted in dark green) and the horticultural pest Epiphyas postvittana (Epos, highlighted in orange) and including Bombyx mori (Bmor) and Ostrinia scapulalis (Osca). Circle at the nodes represent the Shimodaira-Hasegawa local support. Colours and size of circles are proportional to the percentage of support (0-100\%). The clusters shaded in aquamarine highlight biased expression in pheromone glands relative to antennae and clusters shaded in fuchsia indicate biased expression in female antennae relative to pheromone glands

$Z$ and $E$ races of $O$. scapulalis [33]. FAR3 is closely related to OscaFAR II, whereas FAR4 had no similarity with O. scapulalis and B. mori FARs. Distinct from FAR XIII and pgFAR, none of these three FARs were specific to pheromone glands, although the number of counts in the female antennae was extremely low. Two FARs, FAR10 and FAR14, showed biased expression in antennae compared to pheromone glands (Fig. 4). No evidence of positive selection was detected for any of the FAR genes. 


\section{Acetyl-CoA acetyltransferases / 3-ketoacyl-CoA thiolases (ACAT)}

Acetate ester pheromones are produced from the fatty alcohol intermediate by a acetyl-CoA:fatty alcohol acetyltransferase [18]. This enzyme is one of the last steps in the pheromone biosynthetic pathway of many moths, however, to date no pheromone alcohol acetyltransferases has been isolated. Four orthologous groups, with a sequence from each of the five species, contained a conserved domain for acetyl-CoA acetyltransferase (Additional file 16: Figure S5). Except for one group (ACAT4), only partial CDS were recovered from the antennal transcriptomes of E. postvittana and the full CDS became available only after mining the genome. Also, only a partial transcript was recovered for both Ctenopseustis species in the orthologous group ACAT2. All these sequences also contained a thiolase conserved domain and showed similarity to the 3ketoacyl-CoA thiolase, the enzyme that performs the last step of the $\beta$-oxidation cycle. Two genes (ACAT1 and ACAT2) showed pheromone gland biased expression (Additional file 12: Table S9). No evidence of positive selection was observed for any of the four groups.

\section{Acyl-CoA-binding proteins (ACBP)}

ACBPs bind straight-chain (C14-C22) acyl-CoA esters protecting them from hydrolysis [73]. They serve as carriers or cellular deposits for the acyl-CoAs used in pheromone biosynthesis [74]. We identified three orthologous groups with similarity to ACBPs. One group (ACBP1) clustered with the midgut ACBP (mgACBP) of $B$. mori, whereas the pheromone gland ACBP (pgACBP) of $B$. mori clustered with a transcript found only in $P$. excessana (Additional file 17: Figure S6). The sequences forming the two remaining clusters, although showing similarity with ACBPs, were more than 200 amino acids long compared with the approximately 90 amino acids of mgACBPs and pgACBPs. Biased expression was found for ACPB1 but it was not consistent across species. $C$. obliquana showed over expression in antennae, while in the two Planotortrix species the gene was over expressed in the pheromone glands (Additional file 12: Table S9). No evidence of positive selection was observed.

\section{Fatty acid transporter proteins (FATP)}

Fatty acid transport proteins (FATPs) belong to an evolutionary conserved family of membrane-bound proteins that facilitate the uptake of extracellular long chain fatty acids into the cell and catalyze the ATP-dependent esterification of very long-chain fatty acids to the corresponding acyl-CoA derivatives. Twenty-one sequences had a blast hit with very long chain fatty acid transport proteins. The sequences (except one which was excluded from the analysis because it was too short) were clustered into four orthologous groups and their phylogenetic relationships are shown in Additional file 18: Figure S7. The B. mori FATP (NP_00127727), homologous to FATP3, is predominantly expressed in pheromone glands and is up-regulated 1 day prior to eclosion [75]. Although, our moths were collected as adults, FATP3, as well as FATP4, showed biased expression in the pheromone glands of all species except $P$. excessana (FATP3) and P. octo (FATP4) (Additional file 12: Table S9). No evidence of positive selection was observed for any of the FATP genes with any of the models tested, however for the FATP3 we found a positively selected site at position 640 .

\section{Other proteins involved in fatty acid biosynthesis}

Before adult moth emergence, pheromone gland cells produce and accumulate pheromone precursor in the form of triacylglycerols (TAG). In eukaryotes TAGs are synthetized through two major pathways, the glycerol-3phosphate (G3P) and the monoacylglycerol pathways, although in most cells TAG is synthetized mainly by G3P. Triacylglycerol synthesis occurs in the order; glycerol-3phosphate acyltransferase (GPAT; EC 2.3.1.15), 1-acylsn-glycerol-3-phosphate acyltransferase.

(AGPAT; EC 2.3.1.51) and phosphatidate phosphohydrolase (PAP; EC 3.1.3.4) to produce sn-1,2-DAG, a precursor of TAG, phosphatidylcholine and phosphatidylethanolamine [76]. In animals, members of AGPAT have been shown to transfer the unsaturated fatty acyl groups, glycerol-3-phosphate [77]. We found four distinct orthologous groups similar to AGPAT1, 2, 3 and 6 of $H$. virescens. A sequence similar to AGPAT5 was found only in P. octo (Additional file 19: Figure S8). None showed biased expression in the pheromone glands, but one group, AGPAT1, showed biased expression in the antennae compared with pheromone glands (Additional file 12: Table S9). No evidence for positive selection was observed.

Sixty-three sequences from the five species of leafroller moths had a blast hit with elongation of very long chain fatty acids proteins (ELOVL) and were clustered in 14 orthologous groups by ProteinOrtho. After manual curation, we discarded 8 sequences because they were too short or after correction they resulted in transcripts that were identical to others. The phylogenetic analysis revealed 10 clusters, each containing one representative of each species, shown in Additional file 20: Figure S9. No evidence of positive selection was observed for any of these clusters. Two orthologous groups (ELOVL2 and ELOVL1) consistently displayed biased expression in pheromone glands across species (Additional file 12: Table S9).

A total of 106 sequences had similarity with fatty acid transferases (FAT), however 12 of these were excluded from further analysis because they were either too short or contained many missing nucleotides. The remaining 94 
sequences clustered into 23 orthologous groups and their phylogenetic relationships are shown in Additional file 21: Figure S10. Of these groups, 12 were represented in all 5 species. One group (FAT7) showed antennal biased expression relative to pheromone glands in all New Zealand species, whereas FAT19, was antennal biased except in $P$. excessana. FAT17 instead was consistently overexpressed in the pheromone glands across all species (Additional file 12: Table S9). No evidence of positive selection was observed.

A total of 31 sequences had similarity with fatty acid hydrolases (FAAH), four of which were excluded from further analysis because they were only partial. FAAHs belong to the serine hydrolase superfamily and are secondary targets of organophosphates. FAAHs can act as scavenging or detoxifying enzymes and they are principal targets for pest management [78]. FAAH sequences clustered in 5 orthologous groups as shown in the phylogenetic tree in Additional file 22: Figure S11. Sequences belonging to the orthologous groups FAAH1 and FAAH2 were consistently biased in their expression in the pheromone glands of all four New Zealand species (Additional file 12: Table S9). No evidence of positive selection was found.

\section{Odorant receptors}

Odorant receptors (ORs) of the Ctenopseustis and Planotortrix species and of $E$. postvittana have been mined previously $[45,46,49]$ and no new genes have been discovered in this analysis. The biased expression of ORs between male and female antennae was consistent with previous results and only two ORs (OR74 and OR30) were biased in male antennae of all New Zealand endemic moth species (Additional file 12: Table S9, Fig. 7). We are not reporting results on these genes further and direct the reader to the original publications for results. However, OR expression will be discussed in light of all other genes mined in this paper.

\section{Chemosensory proteins and odorant binding proteins}

Chemosensory proteins (CSPs) and Odorant binding proteins (OBPs) are small (10 to $30 \mathrm{kDa})$, globular, water-soluble proteins characterized by four to six highly conserved cysteine residues and the formation of two to three disulphide bonds, respectively [79]. Members of these two classes of protein function as carriers facilitating the transfer of odour molecules to the receptors on the dendrites of olfactory neurons [80-82]. These families are thought to be related, with a common ancestor dating back to after the terrestrialization of the Arthropoda about 380-450 Ma [83]. In contrast to OBPs, which are very diverse and classified into several subfamilies, CSPs are more conserved across insects [84].
A total of 65 sequences in the five leafroller moth species showed similarity with chemosensory proteins and another 23 with ejaculatory-bulb specific proteins. These sequences were clustered into 14 orthologous groups by ProteinOrtho and they were all characterized by the presence of four conserved cysteines typical for chemosensory proteins [85]. Sequences of CSP11 were considerably longer than other CSPs with about 300 residues instead of the typical $\sim 120$ amino acids. After manual correction, 79 sequences remained for which phylogenetic relationships are shown in Additional file 23: Figure S12. CSP4 was most closely related to CSP19 of Sesamia inferens when CSPs from other insects were included in the phylogenetic tree. CSP19 from S. inferens is overexpressed in male antennae and it is thought to play a role in sex pheromone reception [86]. In our moths, none of CSPs showed biased expression in male antennae, whereas CSP1 (OG12340) showed biased expression in female antennae compared to pheromone glands in all New Zealand endemic species (Additional file 12: Table S9, Fig. 4). CSP1 clustered with CSP1, 2 and 3 from the alfalfa plant bug Adelphocoris lineolatus in which these three CSPs are thought to mediate host recognition [87]. In general, CSPs were biased in their expression to pheromone glands (Additional file 12: Table S9, Fig. 4). We found no evidence for positive selection in any CSPs.

We found 209 sequences with similarity to odorant binding protein gene members, including the specific subclass of antennal binding proteins X (ABPX), general odorant binding proteins (GOBP), odorant binding proteins (OBP) and pheromone binding proteins (PBP). Of the 209 contigs, 157 were grouped into 38 orthologous groups, of which 10 were ABPXs, three GOBPs, 13 OBPs and five PBPs. Following the manual curation of contig alignments, we were left with 193 sequences for a total of 44 genes for which phylogenetic relationships are shown in Fig. 8. GOBPs and PBPs are thought to have evolved by a duplication event. They form a monophyletic clade within the OBP gene tree and are typically located in a physical gene cluster [88] (see also Fig. 8). In fact, PBPs and GOBPs form a monophyletic clade with the inclusion of OBP10. The other two subfamilies of odorant binding protein members, ABPXs and OBPs were intermingled and formed four main clusters. One of these contained all odorant binding proteins with extra cysteines and a conserved proline (plus- $\mathrm{C}$ group in Fig. 8). Odorant binding proteins that were missing two conserved cysteines were also clustered together (minus$\mathrm{C}$ group in Fig. 8). Two GOBPs and three PBPs were described in E. postvittana $[49,89]$. In the New Zealand tortricids, we found that both GOBP1 and 2 were duplicated and that a similar duplication event seems to have occurred for each of the PBPs. We identified three genes 


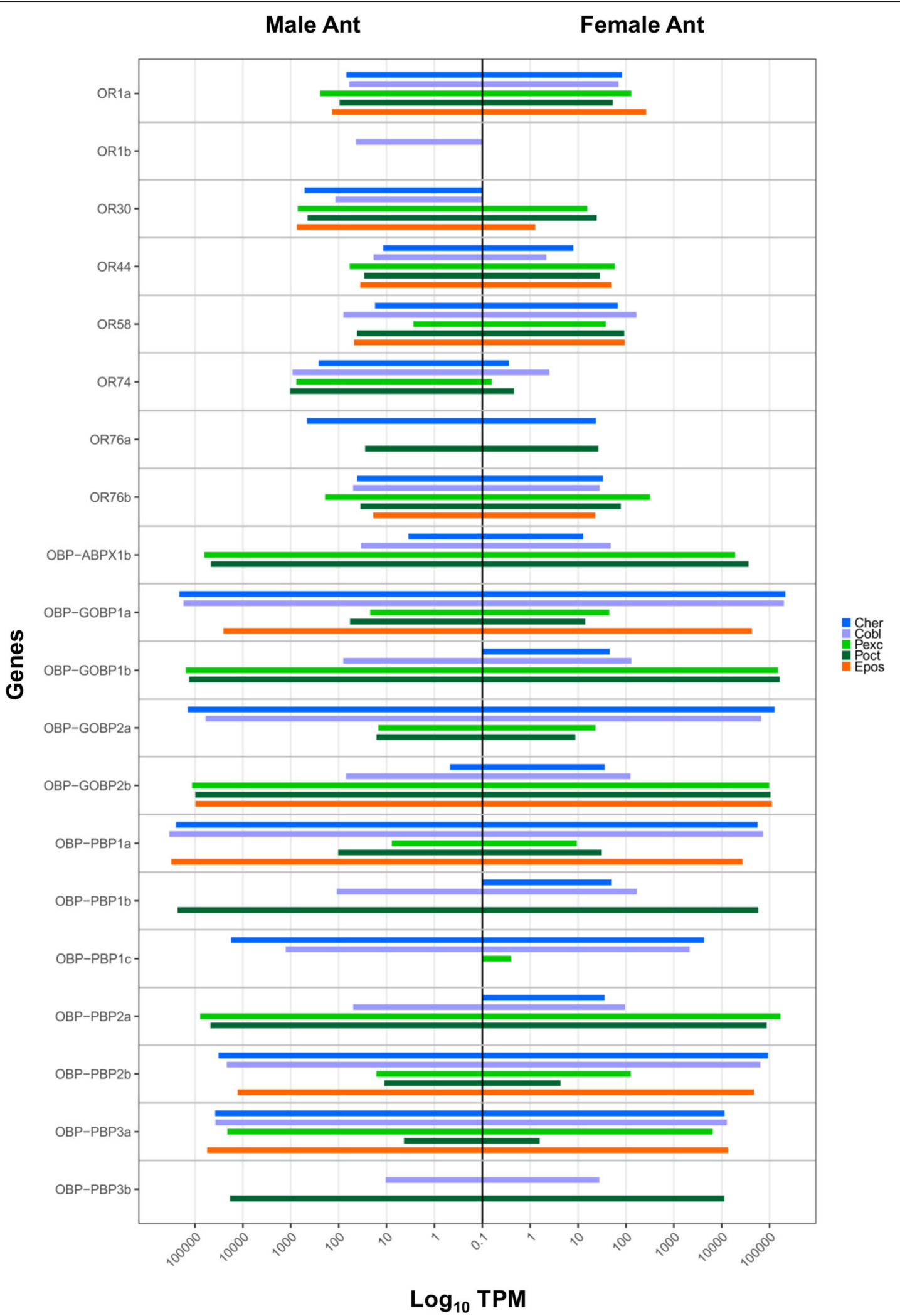

Fig. 7 Expression levels in Transcripts Per Kilobase Million (TPM) in male and female antennae of the genes putatively involved of the peripheral olfactory repertoire in the four New Zealand native leafroller moth species, Ctenopseustis herana, C. obliquana, Planotortrix excessana, and P. octo. Only genes with a log fold change over a threshold of 2 in at least one species are shown 


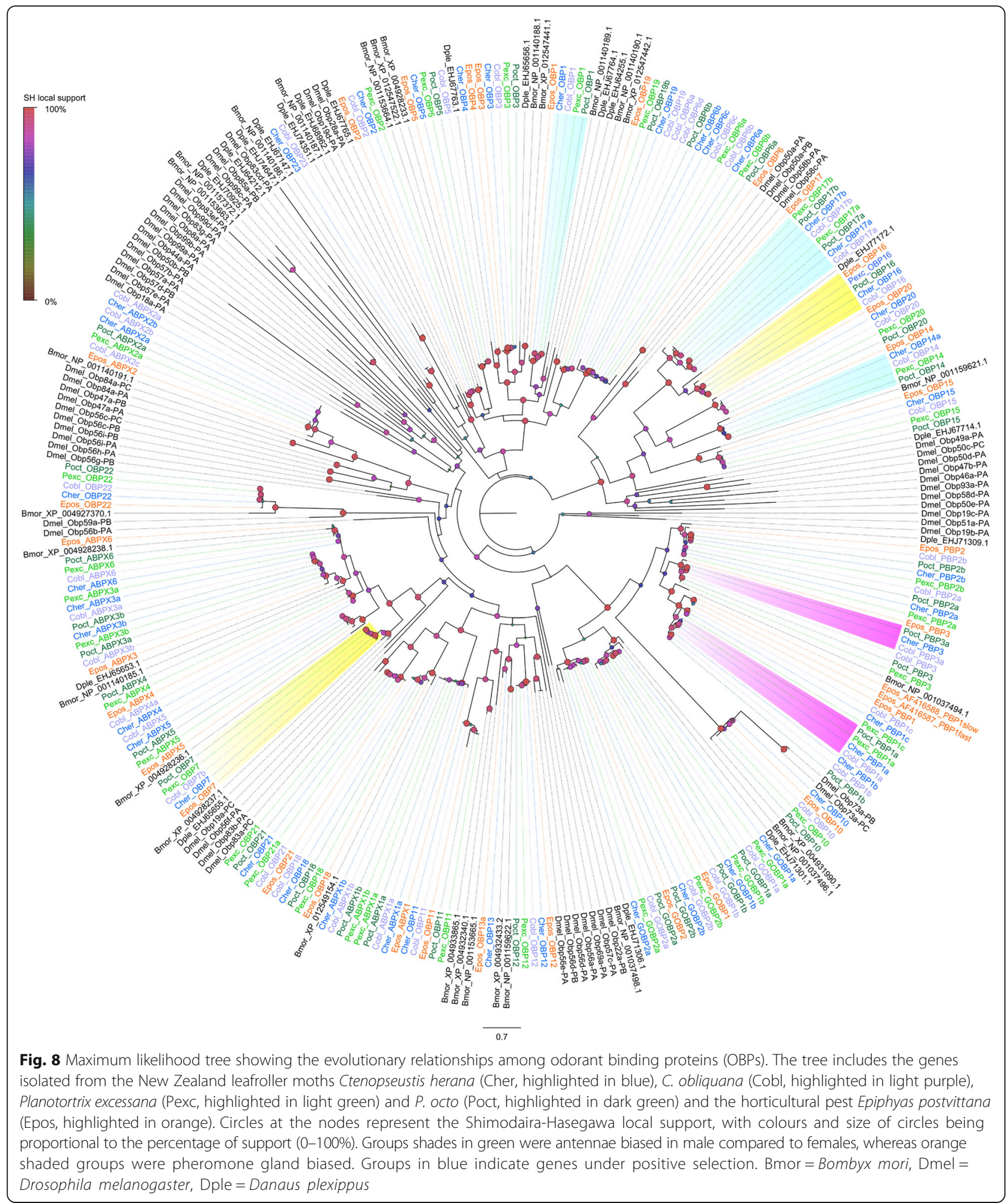

closely related to EposPBP1 (here called them PBP1a, b and c), which differ by more than 70 nucleotide substitutions within a species. These duplications seem to predate the diversification of the two genera, indeed in all the PBP and GOBP orthologous groups the $C$. herana gene is sister to $P$. excessana and the $C$. obliquana representative sister to $P$. octo in the phylogenies. In most of the clusters the expected relationships of genes from co- 
generic species were observed, apart from ABPX3a for which the $C$. herana representative was the sister of $P$. excessana and C. obliquana of $P$. octo.

While GOBPs are generally expressed at similar levels in both male and female antennae, PBPs show strong sexual dimorphism ([90] and references therein). PBP1a and PBP3a were biased in males of the Ctenopseustis species, whereas PBP3b was biased in males of P. excessana. PBP1b and PBP2a were biased in female antennae in C. herana, while PBP1b was male biased in P. octo (Additional file 12: Table S9, Fig. 8). PBP1 and 3 are both capable of binding pheromone components $[89,91]$. The level of expression of the different PBP members was quite different among species (Additional file 12: Table S9, Fig. 7). PBP1a showed the highest expression in Ctenopseustis species whereas, PBP1c was highest in $P$. octo. In P. excessana all three PBP1s were hardly detectable at the transcript level. PBP2a was more highly expressed than PBP2b in both Ctenopseustis species and the opposite was true for the Planotortrix species. A similar expression pattern was observed between the GOBPs and PBP3s, with GOBP1a, GOBP2a and PBP3a highly expressed in Ctenopseustis, whereas GOBP1b, GOBP2b and PBP3b were in Planotortrix. Similarly, another couple of OBPs, ABPX1a and b showed an alternative expression pattern with ABPX1a more highly expressed in Ctenopseustis and ABPX1b in Planotortrix (Additional file 12: Table S9, Fig. 7).

In the expression comparison between female antennae and pheromone glands fourteen groups showed biased expression in the antennae of all species, whereas two plus- $C$ OBPs, OBP14 and OBP17 were pheromone gland biased in their expression (Additional file 12: Table S9, Fig. 4).

Some evidence of positive selection was observed in at least two of three model comparisons for two genes, OBP7 and OBP16, with one significantly selected site in each gene (Additional file 13: Table S10). A significant site was also found in each of ABPX3a and OBP18. PBP1 isolated from several species within Ctenopseustis and Planotortrix revealed rapid rates of sequence evolution along the lineage leading to PBPs used by C. filicis and $C$. fraternal, species that utilize 16 carbon acetates instead of the 14 carbon acetates usually found among member of the two genera $[92,93]$. The tortricids investigated in this study have pheromones composed of 14 carbon acetates and no evidence of positive selection was found for either PBP1 or any other of the PBPs.

\section{Sensory neuron membrane proteins (SNMPs)}

SNMPs are membrane proteins related to the human fatty acid transporter CD36 and are associated with chemosensory neurons in insects. Two classes of SNMPs have been described in insects of which SNMP1 has been shown to be essential for detection of the pheromone cis-vaccenyl acetate in Drosophila [94, 95]. Four orthologous groups, comprising 16 sequences, showed similarity with SNMPs, however OG17722 after visual inspection and manual correction was the same sequence as SNMP2. Of the three remaining groups one showed similarity to SNMP1 with orthologues in all five species and one to Drosophila SNMP2. This latter group had no orthologues in E. postvittana. The last group SNMP3 was sister to SNMP2 and was found in all five leafroller moths but did not have an orthologue in D. melanogaster (Additional file 24: Figure S13). SNMP1 has been shown to be antennal specific in several lepidopteran species $[96,97]$. In the New Zealand tortricids SNMP1 was barely detected in the pheromone glands, and showed higher counts in male antennae compared with females (Additional file 12: Table S9), albeit no significant biased expression was observed. The third group of SNMPs was expressed also in the pheromone glands in contrast to the findings of [98] who did not find SNMPs expressed in pheromone glands (Additional file 12: Table S9). No evidence of positive selection was found for any of these genes.

\section{Ionotropic receptors (IRs)}

The Ionotropic Receptors (IRs) are chemosensory receptors found across all protostomes, forming a highly divergent subfamily of the Ionotropic Glutamate Receptors $[99,100]$. IRs detect volatile acids and ammonia and most are expressed in sensory neurons present in coeloconic sensilla, which do not express odorant receptors [99]. In contrast to ORs, many IRs are conserved in most insects suggesting they comprised the original family of olfactory receptors [100, 101]. A total of 152 sequences had a blast hit with insect IRs and ionotropic glutamate receptors (iGluR). ProteinOrtho clustered 126 of these sequences into 28 orthologues groups. After sequence alignment and manual curation, 120 sequences remained for which the phylogenetic relationships are shown in the Additional file 25: Figure S14. In the phylogenetic analysis we also included Drosophila melanogaster iGluRs and IRs as outgroups. We identify 22 IR genes in the New Zealand tortricid moths compared to the 18 identified in E. postvittana [49] and 63 (including 9 putative pseudogenes) found in D. melanogaster $[99,100]$. In Drosophila 15 IRs are expressed only in antennae and are referred to as antennal IRs [99]. At least 16 clusters of leafroller moth IRs group with the "antennal" IRs of $D$. melanogaster, seven of which were expressed exclusively, or almost exclusively, in the antennae (S Additional file 12: Table S9). Two groups clustered with "divergent" drosophila IRs. These IRs included orthologues of the presumably "ancestral" IR25a of Drosophila, which is expressed in the chemosensory neurons of insects, nematodes and molluscs [100]. This gene displays biased expression in the antennae of the New Zealand tortricids compared to 
pheromone glands (Additional file 12: Table S9) and was most closely related to IR8a, which is thought to be evolved by duplication from IR25a [100]. IR8 also shows biased expression in antennae. Both the IR25a and IR8a genes retain sequence similarity to AMPA iGluRs as shown in the tree (Additional file 25: Figure S14), and are expressed in many coeloconic sensilla where they are thought to act as coreceptors for the more divergent IRs [102]. Four IRs in D. melanogaster are not found in coeloconic sensilla, IR21a, IR40a, IR64a and IR93a, with IR21a expressed in aristal neurons and the other three in sacculus neurons [103]. Arista contain thermosensory and not olfactory neurons, while the sacculus house both olfactory and thermosensory neurons [104]. Neurons expressing IR64a are olfactory, whereas the function of IR40a and IR93a expressing neurons are still unknown [103]. These IRs, except for IR40a, have homologues in the five tortricid species. In contrast to [98] who did not find IRs in the pheromone glands of $A$. ipsilon, two IRs (IR76 and IR25) were also expressed in the pheromone glands in the New Zealand tortricids (Additional file 12: Table S9). Four orthologous groups clustered with iGluR (Additional file 25: Figure S14), two of which, IRiGluR3 and IR-iGluR4, were both biased in their expression in pheromone glands (Additional file 8: Table S8). None of IRs showed evidence of positive selection, although a positive site at alignment position 646, was found in IR-iGluR4 (Additional file 13: Table S10).

\section{Carboxylesterases}

The pheromone signal needs to be degraded so the next signal molecule can be detected by the olfactory sensory neurons (OSNs). Several classes of enzymes are thought to be involved in the degradation of pheromones including cytochrome P450s, GSTs and CXEs, but clear evidence has been found only for CXEs [105]. A total of 322 sequences showed a blast hit (e-values $<2.46 \mathrm{e}^{-11}$ ) with carboxylesterases and 20 with juvenile hormone esterases (JHE). These sequences were grouped into 82 orthologous groups by ProteinOrtho. After inspection of the aligned sequences, manual correction and elimination of the shortest sequences, 210 sequences remained whose phylogenetic relationships are shown in Fig. 9. The carboxylesterase CXE12 of E. postvittana identified by [49] had the best similarity matches to JHEs and had orthologues only in the two Ctenopseustis species. The gene formed a monophyletic group with another JHE (CXE12b) which had an orthologue in all five species. Epiphyas postvittana EposCXE24 (OG513) has been suggested to be the putative carboxylesterase with pheromone degradation ability as it clusters with other pheromone degrading enzymes from other moths [49, 106]. This putative pheromone degrading enzyme forms a monophyletic group with five sequences (CXE24), one from each of the New Zealand endemic leafroller species, except for $C$. herana in which this gene is duplicated. Pheromone degrading enzyme sequences had a best match with the FE4 esterase of the peach potato aphid, Myzus persicae, which is involved in insecticide resistance $[107,108]$. This gene also showed some evidence for positive selection in two out of the three tests and one site under positive selection (Additional file 13: Table S10), but it did not show biased expression (Additional file 12: Table S9) and most likely is ubiquitously expressed as is its orthologue CXE13 of S. littoralis [106]. In three groups of carboxylesterases, CXE6 CXE15 and CXE16, we found multiple paralogues in each of the New Zealand moth species that could indicate a recent expansion. These carboxylesterases have the best matches with the antennal carboxylesterases CXE14, 5 and 10 of Danaus plexippus, S. exigua and S. litura, respectively. CXE15 and two other clusters CXE13 and CXE26 also showed evidence of positive selection in all three model comparisons (Additional file 13: Table S10), however in the case of CXE15 this result should be taken with caution because of the presence of multiple paralogues. Only two carboxylesterases showed biased expression across all four New Zealand species. CXE2b showed biased expression in the pheromone glands whereas CXE27 was biased in the female antennae (Additional file 12: Table S9, Fig. 4). For CXE27 we failed to find an orthologue in E. postvittana. CXE27 clustered with SlitCXE8 which shows biased expression in the antennae in S. littoralis [106]. CXE2, CXE4 and CXE14, although expressed in all three tissues, showed biased expression in female antennae compared to pheromone glands, but there was no difference between male and female antennae (Additional file 12: Table S9). CXE2 and CXE4 lack a signal peptide and cluster with SlitCXE10 and SlitCXE3, both of which belong to the $\alpha$-esterase clade, a group of intracellular esterases that are known for their involvement in the detoxification of insecticides and xenobiotics [109]. CXE14 however contains a signal peptide suggesting it may be secreted into the sensillum lymph [49].

\section{Discussion}

We seek to understand the mode of molecular evolution underpinning sex pheromone evolution in New Zealand's endemic sibling genera Ctenopseustis and Planotortrix. Given that changes in pheromone biosynthesis to produce the different pheromones seem to involve changes in gene expression of desaturase genes [27, 44], we sought to examine whether gene expression evolution is more generally involved in the evolution of pheromone biosynthesis in females and pheromone reception in males. To do this we undertook an analysis 


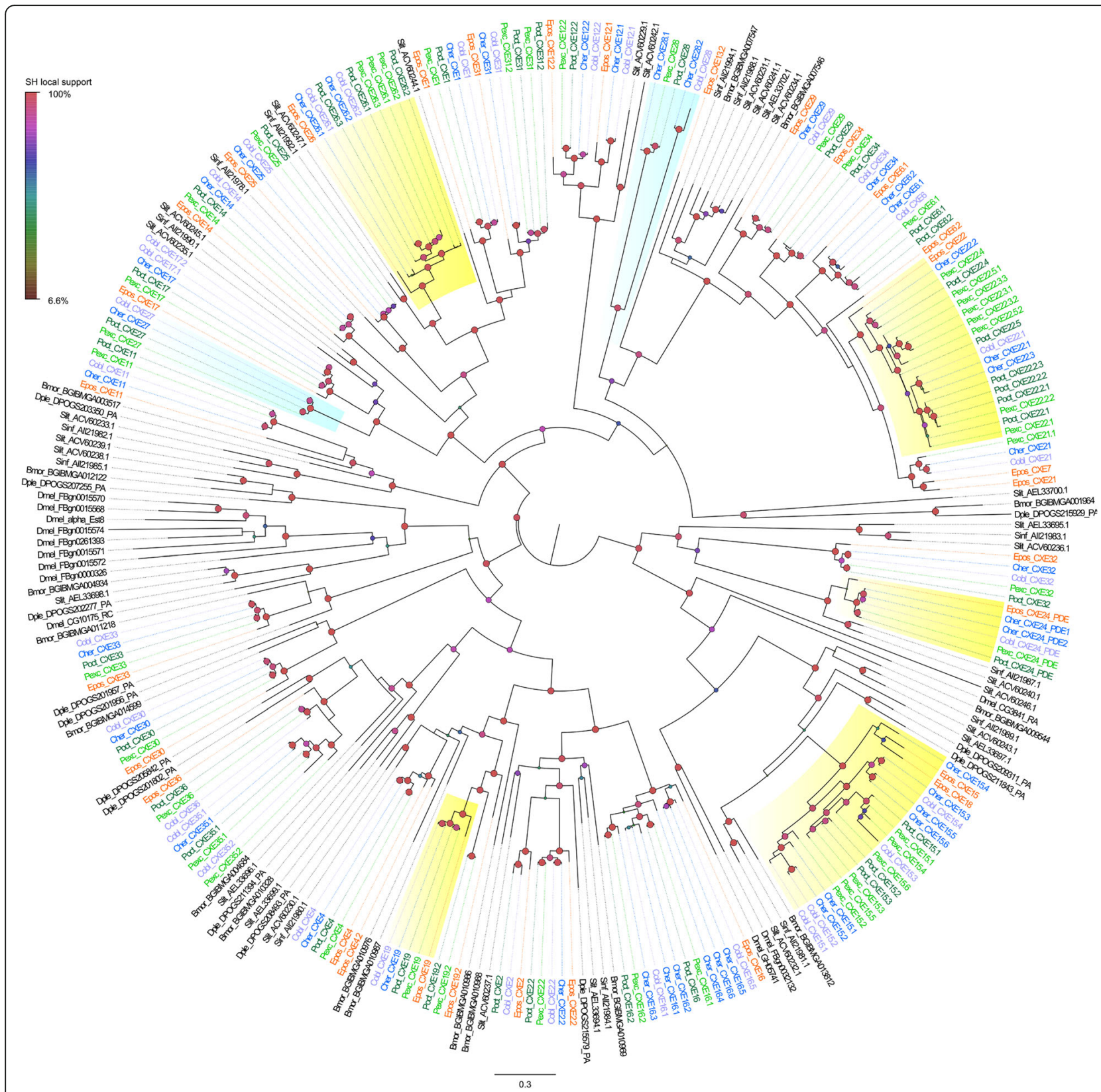

Fig. 9 Maximum likelihood tree showing the evolutionary relationships among carboxylesterases (CXEs). The tree includes the curated genes from the New Zealand leafroller moths Ctenopseustis herana (Cher, highlighted in blue), C. obliquana (Cobl, highlighted in light purple), Planotortrix excessana (Pexc, highlighted in light green) and P. octo (Poct, highlighted in dark green) and the horticultural pest Epiphyas postvittana (Epos, highlighted in orange). Circle at the nodes indicate the Shimodaira-Hasegawa local support, with colour and size of circle being proportional to the percentage of support (0-100\%). Groups shades in orange were pheromone gland biased. Groups in blue indicate positive selection. Bmor = Bombyx mori, Dmel = Drosophila melanogaster, Dple = Danaus plexippus, $\operatorname{Sinf}=$ Sesamia inferens, and Slit = Spodoptera littoralis

of RNAseq data taking both a total transcriptome and candidate gene approach to investigate expression and sequence differences that might underpin the evolution of these mate recognition systems. We identified and manually curated more than 70 new candidate genes in the pheromone biosynthesis and peripheral reception pathways of four closely related species of leafroller moths, Ctenopseustis herana and C. obliquana and
Planotortrix excessana and P. octo. These four species are mostly sympatric and polyphagous, but differ in their pheromone blend (reviewed in [38]). Each species pair are likely to be sibling but this is not completely certain. A fifth tortricid species, Epiphyas postvittana was also included in part of the analyses, however only the antennal transcriptome of both sexes is currently available. The transcriptomes used in this study were the same as 
used by [45, 46, 49], however here we carried out de novo assemblies with several algorithms of all tissues combined for each species. These assemblies were merged within species with Evidentialgene, so the resulting number of final transcripts described here are not directly comparable with those obtained in the previous studies. The newly identified genes from these species such as PBAN and the PBAN receptor, synthases, ACC, $\beta$-oxidation enzymes, FARs, FATs, FATPs, OBPs, CSPs, SNMPs and CXEs have been added to the list of ORs and desaturases already published for these species. Since we did not have biological replicates for each species for the comparison of the expression of these candidate genes we used the closely related species as "biological replicates". We will discuss the commonalities and differences in their expression among species using the RNAseq data. Previous comparisons of differential expression of desaturases and ORs based the RNAseq data on these transcriptomes and quantitative RT-PCR were in very good agreement justifying our comparison using only RNAseq information [27, 45, 46, 49].

The comparison of the transcriptomes of the pheromone glands and expression of mined genes did not reveal new genes thought to be involved in pheromone biosynthesis of these tortricid species. Of all curated candidate genes of the pheromone biosynthetic pathway that could play a role in pheromone differences, we found 11 genes with higher expression in pheromone glands than in antennae in Ctenopseutis and Planotortrix species. These included one $\beta$-oxidation enzyme, six fatty acid reductases, one very long chain fatty acids proteins, two fatty acid hydrolases and one carboxylesterase. Although two of these genes (ACOX3 and FAR2) have been found to be involved in sex pheromone phenotypic variation, $\beta$-oxidases in Agrotis segetum [110] and OscaFAR XIII in Ostrinia scapulalis and pgFAR in B. mori pgFAR which are orthologs of FAR2 [30, 31], none display differences in the expression pattern between species and signature of positive selection that could explain the different pheromones blend of the New Zealand species.

In the pheromone biosynthetic pathways, the only clear differences among these four species relies on the presence of specific desaturases and their differential expression. Ctenopseustis herana, C. obliquana, P. excessana and $P$. octo differ in the use of (Z)-tetradecenyl components of their sex pheromones, with $C$. herana producing Z5-14:OAc exclusively, whereas C. obliquana produces a blend of Z8-14-OAc and Z5-14:OAc, $P$. excessana a blend of Z5-14:OAc and Z7-14:OAc, and $P$. octo almost exclusively producing Z8-14:OAc. These compounds are produced by different desaturases encoded by different genes, which are present in all species (perhaps with the exception of desat7) but are differentially expressed in the pheromone glands. In $P$. octo and C. obliquana Z8-14:OAc is produced by $\Delta 10$ destauration by a desaturase encoded by the desat 5 gene that is down regulated in $C$. herana and $P$. excessana $[27,44]$. The sex pheromone component Z5-14:OAc is part of the blend of both Ctenopseustis species and is produced by a desaturase with $\Delta 5$-destaurase activity encoded by the desat7 gene [71]. This gene is highly expressed in both Ctenopseustis species but no orthologue is expressed in $P$. excessana despite the fact that this species makes use of Z5-14:OAc in its sex pheromone blend. We did not find an orthologue of desat7 in the genome of both $P$. octo and E. postivttana, suggesting that $P$. excessana lacks this gene also. Hagström et al. [71] had shown that Ctenopseustis desat7 clusters distantly from the other classical lepidopteran desaturases. Here we reported several lepidopteran and other insect desaturase genes that could be orthologues of desat7 (Fig. 5) suggesting an ancestral diversification with loss (or loss of function) of the gene in Planotortrix rather than a recent duplication in Ctenopseustis. The lack of desat7 or at least its expression in P. excessana could be due to the biosynthesis of Z5-14:OAc in this species not requiring a specific pheromone gland desaturase. Instead Z5-14:OAc and Z7-14:OAc are most likely biosynthesized from the common monounsaturated fatty acids, oleic and palmitoleic and require two and one cycle of 2-carbon chain-shortening, respectively [70]. A $\Delta 9$-desaturase found in other tissues, other than pheromone glands, is probably involved in the biosynthesis of the oleate and palmitoleate moieties, precursors of Z5-14:OAc [70]. The $\Delta 9$ desaturases are encoded by desat 1 and desat 6 genes, however only the former was found to be expressed in P. excessana and no difference in expression were observed between species [44]. None of the desaturases displayed signatures of positive selection, however we cannot rule out that specific site mutations could be important in explaining the differences in the composition of the sex pheromones among these species. Experiments that manipulate the expression of the desaturases are required to test whether these expression differences are sufficient to generate the altered sex pheromone blend that are observed.

The comparison of male and female antennal transcriptomes revealed that only a few genes have biased expression in male antennae and three of them have some relationship with the pheromone components characteristic of the New Zealand moths. OR74 (OR7 in [45]) and OR30 were the only two genes with male biased expression in all New Zealand species. Three more ORs were male biased in only a single species, OR76a (OR1a in [45]) in C. herana compared with OR1 and OR44 in C. obliquana. These expression results were similar to those obtained with $\mathrm{qPCR}$ in $[45,46]$. OR74 clustered with the pheromone receptors of other 
insects and has been shown to respond to Z8-14:OAc in both Ctenopseustis species. In C. herana OR74 also responded to Z7-14:OAc [45], but interestingly showed the lowest TPM among all species. Instead in C. herana, which exclusively uses Z5-14:OAc as its sex pheromone component, the ORs with the highest TPM were OR30 $(\mathrm{TPM}=513$ and $\operatorname{LogFC}=8.01)$ and a duplicated gene of OR76 (OR76a: TPM 459 and $\operatorname{logFC} 4.46$ ). Only the latter clusters in the sex pheromone receptor clade. OR30 was also highly expressed in $P$. excessana which uses both Z7-14:OAc and Z5-14:OAc as pheromone components, but in this species we found only a single copy of OR76. Despite this relationship between the expression of OR76a and OR30 with Z5-14:OAc, no ORs has been found to respond to Z5-14:OAc in cell-based functional analyses [45]. Recently a QTL-mapping approach in Ostrina nubilalis has revealed that several genes involved in neurogenesis may account for the difference in male response observed between the $E$ and $Z$ strains in addition to ORs [111]. Similar to the Ostrinia strains, the two Ctenopsustis species have swapped the neuronal identity of the pheromone-sensitive neurons, the Z514:OAc-responding OSN has a larger spike amplitude than the Z8-14:OAc-responding OSN in C. herana, whereas in C. obliquana the spike amplitude is reversed [112]. Therefore, a more complex system involving several genes may be operating in the response to different pheromone components in these tortricid moths. In Drosophila expression of ORs and hence the specificity of OSNs can depend on two POU transcription factors, the abnormal chemosensory jump 6 (acj6) and POU domain motif 3 (pdm3), and their interaction $[113,114]$. Both genes are also expressed in the antennae of these tortricid moths and show higher, albeit not significant, expression in male versus female antennae. The expression and functional analysis of these genes needs further investigation.

The fundamental role of OBPs in the reception of odours is supported by several studies which have shown that the majority of these proteins are expressed in the antennae [84]. However, with the discovery of new OBPs by genomics it is becoming clear that many are not associated with the sensory organs and may have very different functions. Our study is not an exception, most of the OBPs were indeed expressed in the antennae. Three OBPs and most of CSPs, showed biased expression in pheromone glands. Two of these three OBPs belonged to the minus-C OBPs, which have been reported to have very broad expression in non-antennal tissues [88]. These "non-olfactory proteins" possess the same structure of olfactory OBPs and CSPs, respectively, making it reasonable to assume that they could be used for the transportation, protection from degradation and release into the environment of hydrophobic molecules [115]. The ability of CSPs to bind sex pheromone has been detected in the pheromone glands of Mamestra brassicae [116], suggesting a role in transporting pheromone for CSPs and OBPs expressed in the pheromone glands.

Odorant binding proteins including PBPs, GOBPs, ABPX3 and OBP18 are all highly expressed in the antennae of both males and females of the New Zealand tortricid moths. PBPs have been shown to solubilize hydrophobic pheromonal compounds, displaying distinct binding specificity [117], interacting differently with different pheromone components [118] and having an effect on the ligand specificity of ORs [119]. Three paralogous PBPs have typically been described from moths, including the close relative to the New Zealand tortricids, E. postvittana [49]. In the New Zealand tortricids, instead we found that all three PBPs seem duplicated and the divergence in all cases seems to predate the divergence of the two genera. In addition to the PBPs, a similar duplication event seems to have occurred for each of the two GOBPs. One possibility is that these different forms represent alleles. Newcomb et al. [89] described two forms of PBP1 in E. postvittana, a fast and a slow form, differing by 18 nucleotide mutations, which through some simple genetics were shown to be alleles of the same locus. In the phylogenetic tree (Fig. 8) the two alleles of EposPBP1 clustered with each other. In the New Zealand tortricids on the other hand, we identified three genes closely related to EposPBP1 (PBP1a, b and $\mathrm{c}$ ), and two for both PBP2 and 3. These forms of PBP found in the New Zealand tortricids do not cluster with each other in the phylogeny containing a high number of substitutional differences and are therefore more likely to be different genes than alleles of the same locus. In other species where high quality genomic information is available, PBPs and GOBPs are often collocated in the genome so perhaps an entire section of the chromosome containing these five genes has been duplicated. These presumably duplicated PBP genes also have distinct expression patterns in male and female antennae, with the PBP1s and PBP3s usually expressed more highly in male antennae and the PBP2s displaying the opposite with higher expression in female antennae. Interestingly the different duplicated PBP genes also show some differences in expression between species. We are not aware of similar results in other moths. Whether this enlarged number of PBPs and differences in their expression between species allows for a greater distinction in binding specificity for different pheromonal compounds remains to be tested. However there are several indications to suggest the important role for PBPs in pheromone reception in these tortricid moths, with PBP1 and 3 having been shown to bind pheromone components in E. postvittana [89] as well as similar male biased expression also observed in E. postvittana [120] 
and in the two Ctenopseustis species. Furthermore, PBP1 from several species within Ctenopseustis and Planotortrix revealed rapid rates of sequence evolution along the lineages leading to those PBPs used by $C$. filicis and $C$. fraternal, species that utilize 16 carbon acetates instead of the 14 carbon acetates usually found among the members of the two genera [92, 93]. In comparison, we did not find clear evidence for positive selection among any of the PBPs. However all the species investigated in this study utilise pheromone components composed exclusively of 14 carbon acetates.

\section{Conclusions}

From the global analyses of antennal and pheromone gland transcriptomes from these New Zealand endemic leafroller moths, only a few genes showing expression differences were identified and similarly when 300 genes involved in pheromone production and reception (including desaturases and ORs) were mined and manually curated, few showed biased expression mirroring the difference in pheromone composition of these species. Furthermore, none of the curated genes showed any clear signs of positive selection at the sequence level. While no clear evidence for selection was found using Codeml, there remains the possibility that sequence differences within some of these genes might confer differences in the mate recognition systems of these species. Certainly differences between species in pheromone biosynthesis seem to be explained predominantly by changes in the expression of two desaturases, desat 5 and desat7, with two genetic factors, one in trans and one in cis, regulating desat5 [44]. More regulatory mutations may be expected to be able to fine tune expression in a set of standing genes in a gene family allowing a more rapid evolution than structural mutations that would impact the specificity of the enzyme [121]. In this way females could rapidly evolve changes in the type and composition of sex pheromones components in their blend. Intraspecific variation in female sex pheromones has been observed in several species both within and between populations [122, 123]. In males, we did not find a clear association between the expression of ORs and sex pheromone reception in the different species, suggesting that other genes could be involved. These tortricid species are characterized by a duplication of their PBP genes, with different paralogs varying in expression in male antennae of the different species. These PBP genes, together with the POU domain transcription factors, could have a role in modulating the specificity of OSNs and need further investigation. In conclusion, this study provides databases and some candidate genes potentially involved in the evolution of new pheromone systems in an enigmatic group of tortricid moths.

\section{Additional files}

Additional file 1: Table S1. Number of raw and cleaned reads for each of mRNA library. (XLSX $9 \mathrm{~kb}$ )

Additional file 2: Table S2. Evidential gene cleansed transcriptomic summary statistics. (XLSX $9 \mathrm{~kb}$ )

Additional file 3: Table S3. Assembly statistics for the different algorithms and different kmer sizes used to assemble the transcriptomes of Ctenopseustis herana, C. obliquana, Planotortrix excessana and P.octo. (XLSX $14 \mathrm{~kb}$ )

Additional file 4: Table S4. Blast description, blast best hit, and GO annotation for each species of tortricid moths used in this work. (XLSX $34301 \mathrm{~kb})$

Additional file 5: Figure S1. Gene Ontology (GO) classifications of species of New Zealand tortricid moths according to biological processes, cellular component and molecular function. (TIFF $1218 \mathrm{~kb}$ )

Additional file 6: Table S5. List of groups of orthology obtained with ProteinOrtho. (XLSX $1587 \mathrm{~kb})$

Additional file 7: Table S6. List of genes with significant bias expression in the comparison of sex antennae and between female antennae and pheromone glands. (XLSX $62 \mathrm{~kb}$ )

Additional file 8: Table S7. Gene set enrichment analyses from the comparison of male and female antennae gene expression. GSA was obtained with Piano using the statistical method "mean". GO terms were used to define gene sets. (XLSX $3609 \mathrm{~kb}$ )

Additional file 9: Figure S2. Consensus heatmaps of the GSA analyses of male and female antennae showing consensus scores, based on rank aggregation, for each directionality class (up $=$ up regulated genes in male antennae, down = up regulated genes in female antennae). (TIFF $1567 \mathrm{~kb})$

Additional file 10: Table S8. Gene set enrichment analyses from the comparison of female antennae and pheromone glands gene expression. GSA was obtained with Piano using the statistical method "mean". GO terms were used to define gene sets. (XLSX $3169 \mathrm{~kb}$ )

Additional file 11: Figure S3. Consensus heatmaps of the GSA analyses of female antennae and pheromone glands showing consensus scores, based on rank aggregation, for each directionality class (up = up regulated genes in female antennae, down = up regulated genes in pheromone glands). (TIFF $2099 \mathrm{~kb})$

Additional file 12:Table S9. List of manually curated genes and their expression values in the different tissues and species. (XLSX $231 \mathrm{~kb}$ )

Additional file 13: Table S10. Positive selection results on the manually curated genes. (XLSX $30 \mathrm{~kb}$

Additional file 14: Nucleotide sequences of the manually mined genes. (DOCX $447 \mathrm{~kb}$ )

Additional file 15: Figure S4. Maximum likelihood tree showing the evolutionary relationships among acyl-CoA oxidase (ACOX) proteins mined from the transcriptomes of the New Zealand leafroller moths Ctenopseustis herana (Cher, highlighted in blue), C. obliquana (Cobl, highlighted in light purple), Planotortrix excessana (Pexc, highlighted in light green) and P. octo (Poct, highlighted in dark green) and the horticultural pest Epiphyas postvittana (Epos, highlighted in orange). Number at the nodes represent the Shimodaira-Hasegawa local support. The cluster (ACOX3a) shaded in orange showed bias expression in pheromone glands relative to antennae. (TIFF $301 \mathrm{~kb}$ )

Additional file 16: Figure S5. Maximum likelihood tree showing the evolutionary relationships among acetyl-CoA acetyltransferase (ACAT) proteins mined from the transcriptomes of the New Zealand leafroller moths Ctenopseustis herana (Cher, highlighted in blue), C. obliquana (Cobl, highlighted in light purple), Planotortrix excessana (Pexc, highlighted in light green) and P. octo (Poct, highlighted in dark green) and the horticultural pest Epiphyas postvittana (Epos, highlighted in orange). Circle size and colour at the nodes represent the ShimodairaHasegawa local support. Aips $=$ Agrotis ipsilum, Bmor $=$ Bombyx mori, Dmel = Drosophila melanogaster, Dple = Danaus plexippus, Hvir = Heliothis 
virescens, Hsub $=$ H. subflexa, Osca $=$ Ostrinia scapulalis and Pxyl $=$ Plutella xylostella. (TIFF $431 \mathrm{~kb}$ )

Additional file 17: Figure S6. Maximum likelihood tree showing the evolutionary relationships among Acyl-CoA-binding proteins (ACBP) mined from the transcriptomes of the New Zealand leafroller moths Ctenopseustis herana (Cher, highlighted in blue), C. obliquana (Cobl, highlighted in light purple), Planotortrix excessana (Pexc, highlighted in light green) and P. octo (Poct, highlighted in dark green) and the horticultural pest Epiphyas postvittana (Epos, highlighted in orange). Circle size and colour at the nodes represent the Shimodaira-Hasegawa local support. Bmor $=$ Bombyx mori, Dmel = Drosophila melanogaster, Dple $=$ Danaus plexippus, Harm $=$ Helicoverpa armigera and Pxyl $=$ Plutella xylostella. (TIFF $430 \mathrm{~kb}$ )

Additional file 18: Figure S7. Maximum likelihood tree showing the evolutionary relationships among fatty acid transporter proteins (FATP). The tree includes the genes isolated from the New Zealand leafroller moths Ctenopseustis herana (Cher, highlighted in blue), C. obliquana (Cobl, highlighted in light purple), Planotortrix excessana (Pexc, highlighted in light green) and P. octo (Poct, highlighted in dark green) and the horticultural pest Epiphyas postvittana (Epos, highlighted in orange). Circle size and colour at the nodes represent the Shimodaira-Hasegawa local support. The clusters shaded in orange showed bias expression in PGs relative to antennae, whereas that in green showed bias in female antennae compared to PG. Bmor = Bombyx mori, Dmel = Drosophila melanogaster Dple $=$ Danaus plexippus and Pxyl = Plutella xylostella. (TIFF $450 \mathrm{~kb}$ )

Additional file 19: Figure S8. Maximum likelihood tree showing the evolutionary relationships among 1-acyl-sn-glycerol-3-phosphate acyltransferase (AGPAT). The tree includes the genes isolated from the New Zealand leafroller moths Ctenopseustis herana (Cher, highlighted in blue), C. obliquana (Cobl, highlighted in light purple), Planotortrix excessana (Pexc, highlighted in light green) and P. octo (Poct, highlighted in dark green) and the horticultural pest Epiphyas postvittana (Epos, highlighted in orange). Circle size and colour at the nodes represent the Shimodaira-Hasegawa local support. The cluster shaded in orange showed bias expression in PGs relative to antennae, whereas that in green showed bias in female antennae compared to PG. Bmor = Bombyx mori, Dmel = Drosophila melanogaster, Dple $=$ Danaus plexippus, Hvir $=$ Heliothis virescens and Pxyl $=$ Plutella xylostella. (TIFF $473 \mathrm{~kb}$ )

Additional file 20: Figure S9. Maximum likelihood tree showing the evolutionary relationships among elongation of very long chain fatty acids proteins (ELOVL). The tree includes the genes isolated from the New Zealand leafroller moths Ctenopseustis herana (Cher, highlighted in blue), C. obliquana (Cobl, highlighted in light purple), Planotortrix excessana (Pexc, highlighted in light green) and P. octo (Poct, highlighted in dark green) and the horticultural pest Epiphyas postvittana (Epos, highlighted in orange). Circle size and colour at the nodes represent the Shimodaira-Hasegawa local support. Bmor $=$ Bombyx mori, Dmel = Drosophila melanogaster, Dple = Danaus plexippus and Pxyl = Plutella xylostella. (TIFF $585 \mathrm{~kb}$ )

Additional file 21: Figure S10. Maximum likelihood tree showing the evolutionary relationships among fatty acid transferases (FAT). The tree includes the genes isolated from the New Zealand leafroller moths Ctenopseustis herana (Cher, highlighted in blue), C. obliquana (Cobl, highlighted in light purple), Planotortrix excessana (Pexc, highlighted in light green) and P. octo (Poct, highlighted in dark green) and the horticultural pest Epiphyas postvittana (Epos, highlighted in orange). Circle size and colour at the nodes represent the Shimodaira-Hasegawa local support. The clusters shaded in orange showed bias expression in pheromone glands relative to antennae, whereas those shaded in green showed bias in female antennae relative to pheromone glands. Aips = Agrotis ipsilon, Bmor $=$ Bombyx mori, Dmel = Drosophila melanogaster, Dple $=$ Danaus plexippus, Hvir $=$ Heliothis virescens, Osca = Ostrinia scapulalis and Pxyl = Plutella xylostella. (TIFF $906 \mathrm{~kb}$ )

Additional file 22: Figure S11. Maximum likelihood tree showing the evolutionary relationships among fatty acid hydrolases (FAAH). The tree includes the genes isolated from the New Zealand leafroller moths Ctenopseustis herana (Cher, highlighted in blue), C. obliquana (Cobl, highlighted in light purple), Planotortrix excessana (Pexc, highlighted in light green) and P. octo (Poct, highlighted in dark green) and the horticultural pest Epiphyas postvittana (Epos, highlighted in orange). Circle size and colour at the nodes represent the Shimodaira-Hasegawa local support. Bmor $=$ Bombyx mori, Dmel $=$ Drosophila melanogaster, Dple $=$ Danaus plexippus, and Pxyl = Plutella xylostella. (TIFF $433 \mathrm{~kb}$ )

Additional file 23: Figure S12. Maximum likelihood tree showing the evolutionary relationships among chemosensory proteins (CSPs). The tree includes the genes isolated from the New Zealand leafroller moths Ctenopseustis herana (Cher, highlighted in blue), C. obliquana (Cobl, highlighted in light purple), Planotortrix excessana (Pexc, highlighted in light green) and P. octo (Poct, highlighted in dark green) and the horticultural pest Epiphyas postvittana (Epos, highlighted in orange). Circle at the nodes represent the Shimodaira-Hasegawa local support. Colours and size of circles are proportional to the percentage of support (0-100\%). Groups shades in orange were pheromone gland biased. Bmor $=$ Bombyx mori, Dmel = Drosophila melanogaster, Harm = Helicoverpa armigera, Hvir = Heliothis virescen and Pxyl = Plutella xylostella. (TIFF $968 \mathrm{~kb}$ )

Additional file 24: Figure S13. Maximum likelihood tree showing the evolutionary relationships among sensory neuron membrane proteins (SNMPs). The tree also includes the CD36 genes of Drosophila melanogaster (Dmel). Numbers at the nodes represent the Shimodaira-Hasegawa local support. Cher $=$ Ctenopseustis herana (highlighted in blue), Cobl =C. obliquana (highlighted in light purple), Pexc $=$ Planotortrix excessana (highlighted in light green), Poct $=P$. octo (highlighted in dark green), Epos = Epiphyas postvittana (highlighted in orange), Aips = Agrotis ipsilum, Bmor $=$ Bombyx mori, Dple = Danaus plexippus, Hvir = Heliothis virescens, Pxyl = Plutella xylostella. (TIFF $486 \mathrm{~kb})$

Additional file 25: Figure S14. Maximum likelihood tree showing the evolutionary relationships among ionotropic receptors (IRs). The tree includes the genes isolated from the New Zealand leafroller moths Ctenopseustis herana (Cher, highlighted in blue), C. obliquana (Cobl, highlighted in light purple), Planotortrix excessana (Pexc, highlighted in light green) and P. octo (Poct, highlighted in dark green) and the horticultural pest Epiphyas postvittana (Epos, highlighted in orange). Circle at the nodes represent the Shimodaira-Hasegawa local support. Colour and size of circle is proportional to the percentage of support (0-100\%). Groups shades in orange were pheromone gland biased. Bmor $=$ Bombyx mori and Dmel = Drosophila melanogaster. (TIFF $1441 \mathrm{~kb}$ )

\section{Abbreviations}

ACBP: Acyl-CoA-binding protein; ACC: Acetyl-CoA carboxylase; ACOX: AcylCoA oxidase; AGPAT: 1-acyl-sn-glycerol-3-phosphate acyltransferase; AMPA: a-amino-3-hydroxy-5-methyl-4-isoxazolepropionic acid receptor; CSP: Chemosensory protein; CXE: Carboxylesterase; ELOVL: Very long chain fatty acids proteins; FAAH: Fatty acid amide hydrolase; FAR: Fatty acid reductase; FAS: Fatty acid synthase; FAT: Fatty acid transferase; FATP: Fatty acid transport protein; GO: Gene Ontology; GOBP: General odorant binding protein; GSA: Gene Set Analysis; IR: Ionotropic receptor; JHE: Juvenile hormone esterase; LRT: Likelihood ratio test; OBP: Odorant binding protein; OSN: Olfactory sensory neuron; PBAN: Pheromone biosynthesis activating neuropeptide; PBP: Pheromone binding protein; PG: Pheromone glands; SNMP: Sensory neuron membrane protein; TPM: Transcripts Per Kilobase Million

\section{Acknowledgments}

We would like to thanks Melissa Jordan for providing previously mined genes, Jérôme Albre for dissection of pheromone glands, Mark Wholers for figure preparation and Anne Barrington for moth rearing. Finally, we thank two anonymous reviewers for their valuable comments on the previous version of the manuscript.

\section{Funding}

This research was funded by the MIUR (Ex-60\% 2016) to AG and funds from The Alan Wilson Centre to RDN.

\section{Availability of data and materials}

All data used in this study have been made publicly available (see Methods for NCBI accession numbers) or included in this article and its supplementary files. 


\section{Authors' contributions}

AG and RDN conceptualised the study and drafted the manuscript. BS and AHT developed the transcriptome databases and performed the bioinformatic analyses. All authors read and approved the final manuscript.

\section{Ethics approval and consent to participate}

No ethics approval and consent to participate was required for the collection or experimentation of the insect species used in this study, namely Ctenopseustis herana, C. obliquana, Planotortrix excessana, P. octo and Epiphyas postvittana. This study did not involve endangered species.

\section{Consent for publication}

Not applicable

\section{Competing interests}

The authors declare that they have no competing interests.

\section{Publisher's Note}

Springer Nature remains neutral with regard to jurisdictional claims in published maps and institutional affiliations.

\section{Author details}

'Department of Biology, University of Padova, Padova, Italy. ${ }^{2}$ The New Zealand Institute for Plant \& Food Research Ltd, Auckland, New Zealand. ${ }^{3}$ School of Biological Sciences, University of Auckland, Auckland, New Zealand.

Received: 13 October 2017 Accepted: 14 January 2018

Published online: 26 January 2018

\section{References}

1. Lassance J-M, Löfstedt C. Concerted evolution of male and female display traits in the European corn borer, Ostrinia nubilalis. BMC Biol. 2009;7:10

2. Groot AT, Dekker T, Heckel DG. The genetic basis of pheromone evolution in moths. Annu Rev Entomol. 2015 https://doi.org/10.1146/annurev-ento010715-023638.

3. Symonds MRE, Elgar MA. The evolution of pheromone diversity. Trends Ecol Evol. 2008:23:220-8

4. Phelan PL. Evolution of sex pheromones and the role of asymmetric tracking. In: Roitberg BD, Isman MB, editors. Insect chemical ecology: an evolutionary approach. New York: Chapman \& Hall; 1992. p. 265-314.

5. Harari AR, Steinitz H. The evolution of female sex pheromones. Curr Zool. 2013;59:569-78.

6. Carroll SB. Evolution at two levels: on genes and form. PLoS Biol. 2005; 3:e245.

7. King MC, Wilson AC. Evolution at two levels in humans and chimpanzees. Science. 1975:188:107-16.

8. Loire E, Tusso S, Caminade P, Severac D, Boursot P, Ganem G, et al. Do changes in gene expression contribute to sexual isolation and reinforcement in the house mouse? Mol Ecol. 2017; https://doi.org/10.1111/ mec.14212.

9. Wolf JBW, Bayer T, Haubold B, Schilhabel M, Rosenstiel P, Tautz D. Nucleotide divergence vs. gene expression differentiation: comparative transcriptome sequencing in natural isolates from the carrion crow and its hybrid zone with the hooded crow. Mol Ecol. 2010;19:162-75.

10. Ellegren $\mathrm{H}$, Parsch J. The evolution of sex-biased genes and sex-biased gene expression. Nat Rev Genet. 2007;8:689-98.

11. Hoang K, Matzkin LM, Bono JM. Transcriptional variation associated with cactus host plant adaptation in Drosophila mettleri populations. Mol Ecol. 2015;24:5186-99.

12. Bailey Rl, Innocenti P, Morrow EH, Friberg U, Qvarnström A. Female Drosophila melanogaster gene expression and mate choice: the $X$ chromosome harbours candidate genes underlying sexual isolation. PLoS One. 2011;6:e17358.

13. Cassone BJ, Mouline K, Hahn MW, White BJ, Pombi M, Simard F, et al. Differential gene expression in incipient species of Anopheles gambiae. Mol Ecol. 2008;17:2491-504

14. Fujii T, Fujii T, Namiki S, Abe H, Sakurai T, Ohnuma A, et al. Sex-linked transcription factor involved in a shift of sex-pheromone preference in the silkmoth Bombyx mori. Proc Natl Acad Sci. 2011;108:18038-43.
15. Löfstedt C. Population variation and genetic control of pheromone communication systems in moths. Entomol Exp Appl. 1990:54:199-218.

16. Bjostad LB, Wolf WA, Roelofs WL. Pheromone biosynthesis in lepidopterans: desaturation and chain shortening. In: Prestwich GD, Blomquist GJ, editors. Pheromone biochemistry. New York: Academic Press; 1987. p. 77-120.

17. Roelofs WL, Wolf WA. Pheromone biosynthesis in lepidoptera. J Chem Ecol. 1988;14:2019-31.

18. Jurenka R. Insect pheromone biosynthesis. In: Schulz S, editor. The chemistry of pheromones and other semiochemicals I. Berlin: Springer; 2004. p. 97132. https://doi.org/10.1007/b95450.

19. Bellas TE, Bartell RJ, Hill A. Identification of two components of the sex pheromone of the moth, Epiphyas postvittana (Lepidoptera, Tortricidae). J Chem Ecol. 1983:9:503-12.

20. Löfstedt C, Bengtsson M. Sex pheromone biosynthesis of $(E, E)-8,10-$ dodecadienol in codling moth Cydia pomonella involvesE9 desaturation. J Chem Ecol. 1988;14:903-15.

21. Knipple DC, Rosenfield C-L, Miller SJ, Liu W, Tang J, Ma PWK, et al. Cloning and functional expression of a cDNA encoding a pheromone gland-specific acyl-CoA $\Delta 11$-desaturase of the cabbage looper moth, Trichoplusia Ni. Proc Natl Acad Sci. 1998;95:15287-92.

22. Liu W, Ma PWK, Marsella-Herrick P, Rosenfield C-L, Knipple DC, Roelofs W. Cloning and functional expression of a cDNA encoding a metabolic acylCoA $\Delta$ 9-desaturase of the cabbage looper moth, Trichoplusia ni. Insect Biochem Mol Biol. 1999:29:435-43.

23. Roelofs WL, Liu W, Hao G, Jiao H, Rooney AP, Linn CE. Evolution of moth sex pheromones via ancestral genes. Proc Natl Acad Sci. 2002;99:13621-6.

24. Matoušková P, Pichová I, Svatoš A. Functional characterization of a desaturase from the tobacco hornworm moth (Manduca sexta) with bifunctional Z11- and 10,12-desaturase activity. Insect Biochem Mol Biol. 2007:37:601-10.

25. Liénard MA, Strandh M, Hedenström E, Johansson T, Löfstedt C. Key biosynthetic gene subfamily recruited for pheromone production prior to the extensive radiation of Lepidoptera. BMC Evol Biol. 2008;8:270.

26. Vogel H, Heidel AJ, Heckel DG, Groot AT. Transcriptome analysis of the sex pheromone gland of the noctuid moth Heliothis virescens. BMC Genomics. 2010;11:29.

27. Albre J, Liénard MA, Sirey TM, Schmidt S, Tooman LK, Carraher C, et al. Sex pheromone evolution is associated with differential regulation of the same desaturase gene in two genera of leafroller moths. PLOS Genet. 2012;8:e1002489.

28. Köblös G, Dankó T, Sipos K, Geiger Á, Szlanka T, Fodor J, et al. The regulation of $\Delta 11$-desaturase gene expression in the pheromone gland of Mamestra brassicae (Lepidoptera; Noctuidae) during pheromonogenesis. Gen Comp Endocrinol. 2015:221:217-27.

29. Buček A, Matoušková P, Vogel H, Šebesta P, Jahn U, Weißflog J, et al. Evolution of moth sex pheromone composition by a single amino acid substitution in a fatty acid desaturase. Proc Natl Acad Sci. 2015;112:12586-91.

30. Moto K, Yoshiga T, Yamamoto M, Takahashi S, Okano K, Ando T, et al. Pheromone gland-specific fatty-acyl reductase of the silkmoth, Bombyx mori. Proc Natl Acad Sci. 2003:100:9156-61.

31. Antony B, Fujii T, Moto K, Matsumoto S, Fukuzawa M, Nakano R, et al. Pheromone-gland-specific fatty-acyl reductase in the adzuki bean borer, Ostrinia scapulalis (Lepidoptera: Crambidae). Insect Biochem Mol Biol. 2009;39:90-5.

32. Liénard MA, Hagström ÅK, Lassance J-M, Löfstedt C. Evolution of multicomponent pheromone signals in small ermine moths involves a single fatty-acyl reductase gene. Proc Natl Acad Sci. 2010;107:10955-60.

33. Lassance J-M, Groot AT, Liénard MA, Antony B, Borgwardt C, Andersson F, et al. Allelic variation in a fatty-acyl reductase gene causes divergence in moth sex pheromones. Nature. 2010;466:486-9.

34. Hagström ÅK, Liénard MA, Groot AT, Hedenström E, Löfstedt C. Semiselective fatty acyl reductases from four heliothine moths influence the specific pheromone composition. PLoS One. 2012;7:e37230.

35. Leal WS. Odorant reception in insects: roles of receptors, binding proteins, and degrading enzymes. Annu Rev Entomol. 2013;58:373-91.

36. Leary GP, Allen JE, Bunger PL, Luginbill JB, Linn CE, Macallister IE, et al. Single mutation to a sex pheromone receptor provides adaptive specificity between closely related moth species. Proc Natl Acad Sci. 2012;109:14081-6

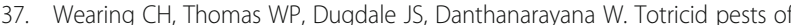
Pome and stone fruits, Australian and New Zealand species. In: van der Geest LPS, Evenhuis HH, editors. Tortricid pests their biology, natural enemies and control. Amsterdam: Elsevier Science; 1991. 
38. Newcomb RD, Steinwnder B, Albre J, Foster SP. The endemic New Zealand genera Ctenopseustis and Planotortrix: a down-under story of leafroller moth sex pheromone evolution and speciation. In: Allison JD, Cardé RT, editors. Pheromone communication in moths: evolution, behavior and application. Los Angeles: University of California Press; 2016. p. 277-90.

39. Foster SP, Roelofs WL. Sex pheromone differences in populations of the brownheaded leafroller, Ctenopseustis obliquana. J Chem Ecol. 1987;13:623-9.

40. Galbreath RA, Benn MH, Young H, Holt VA. Sex pheromone components in the New Zealand greenheaded leafroller. Planotortrix excessana (Lepidoptera: Tortricidae). Z Für Naturforschung C. 1985;40:266-71.

41. Foster SP, Clearwater JR, Muggleston SJ, Shaw PW. Sex pheromone of a Planotortrix excessana sibling species and reinvestigation of related species. J Chem Ecol. 1990;16:2461-74.

42. Young $H$, Galbreath RA, Benn MH, Holt VA, Struble DL. Sex pheromone components in the New Zealand brownheaded leafroller Ctenopseustis obliquana (Lepidoptera: Tortricidae). Z Für Naturforschung C. 1985:40:262-5.

43. Foster SP, Clearwater JR, Muggleston SJ, Dugdale JS, Roelofs WL. Probable sibling species complexes within two described New Zealand leafroller moths. Naturwissenschaften. 1986;73:156-8.

44. Albre J, Steinwender B, Newcomb RD. The evolution of desaturase gene regulation involved in sex pheromone production in leafroller moths of the genus Planotortrix. J Hered. 2013;104:627-38.

45. Steinwender B, Thrimawithana AH, Crowhurst RN, Newcomb RD. Pheromone receptor evolution in the cryptic leafroller species, Ctenopseustis obliquana and C. herana. J Mol Evol. 2014;80:42-56.

46. Steinwender B, Thrimawithana AH, Crowhurst R, Newcomb RD. Odorant receptors of the New Zealand endemic leafroller moth species Planotortrix octo and P. excessana. PLoS One. 2016;11:e0152147.

47. Gleeson D, Holder P, Newcomb R, Howitt R, Dugdale J. Molecular phylogenetics of leafrollers: application to DNA diagnostics. N Z Plant Prot. 2000;53:157-62.

48. Jordan MD, Anderson A, Begum D, Carraher C, Authier A, Marshall SDG, et al. Odorant receptors from the light brown apple moth (Epiphyas postvittana) recognize important volatile compounds produced by plants. Chem Senses. 2009;34:383-94.

49. Corcoran JA, Jordan MD, Thrimawithana AH, Crowhurst RN, Newcomb RD. The peripheral olfactory repertoire of the lightbrown apple moth, Epiphyas postvittana. PLoS ONE. 2015;10:e0128596.

50. Babraham Bioinformatics - FastQC A Quality Control tool for High Throughput Sequence Data. https://www.bioinformatics.babraham.ac.uk/ projects/fastqc/. Accessed 18 Jan 2018.

51. Langmead B, Trapnell C, Pop M, Salzberg SL. Ultrafast and memory-efficient alignment of short DNA sequences to the human genome. Genome Biol. 2009;10:R25.

52. Aronesty E. Comparison of sequencing utility programs. Open Bioinforma J. 2013;7:1-8.

53. Wood DE, Salzberg SL. Kraken: ultrafast metagenomic sequence classification using exact alignments. Genome Biol. 2014;15:R46.

54. Xie Y, Wu G, Tang J, Luo R, Patterson J, Liu S, et al. SOAPdenovo-trans: de novo transcriptome assembly with short RNA-Seq reads. Bioinformatics. 2014;30:1660-6.

55. Grabherr MG, Haas BJ, Yassour M, Levin JZ, Thompson DA, Amit I, et al. Fulllength transcriptome assembly from RNA-Seq data without a reference genome. Nat Biotechnol. 2011;29:644-52.

56. Haas BJ, Delcher AL, Mount SM, Wortman JR, Jr RKS, Hannick LI, et al. Improving the Arabidopsis genome annotation using maximal transcript alignment assemblies. Nucleic Acids Res. 2003;31:5654-66.

57. Gilbert D. EvidentialGene: mRNA Transcript Assembly Software. http:// arthropods.eugenes.org/EvidentialGene/about/EvidentialGene_trassembly_ pipe.html. Accessed 18 Jan 2018.

58. Lechner M, Findeiß S, Steiner L, Marz M, Stadler PF, Prohaska SJ. Proteinortho: detection of (co-)orthologs in large-scale analysis. BMC Bioinformatics. 2011;12:124.

59. Altschul SF, Madden TL, Schaffer AA, Zhang J, Zhao JZ. Gapped BLAST and PSI-BLAST: a new generation of protein database search programs. Nucleic Acids Res. 1997:25:3389-402.

60. Conesa A, Götz S, García-Gómez JM, Terol J, Talón M, Robles M. Blast2GO: a universal tool for annotation, visualization and analysis in functional genomics research. Bioinformatics. 2005;21:3674-6.

61. Larkin MA, Blackshields G, Brown NP, Chenna R, McGettigan PA, McWilliam $H$, et al. Clustal W and Clustal X version 2.0. Bioinformatics. 2007;23:2947-8.
62. Price MN, Dehal PS, Arkin AP. FastTree 2 - approximately maximumlikelihood trees for large alignments. PLoS One. 2010;5:e9490.

63. Yang Z. PAML: a program package for phylogenetic analysis by maximum likelihood. CABIOS. 1997:13:555-6.

64. Langmead B, Salzberg SL. Fast gapped-read alignment with bowtie 2. Nat Methods. 2012;9:357-9.

65. Anders S, Pyl PT, Huber W. HTSeq-a python framework to work with highthroughput sequencing data. Bioinformatics. 2015:31:166-9.

66. Love Ml, Huber W, Anders S. Moderated estimation of fold change and dispersion for RNA-seq data with DESeq2. Genome Biol. 2014;15:550.

67. Väremo L, Nielsen J, Nookaew I. Enriching the gene set analysis of genomewide data by incorporating directionality of gene expression and combining statistical hypotheses and methods. Nucleic Acids Res. 2013;41:4378-91

68. Smith S, Witkowski A, Joshi AK. Structural and functional organization of the animal fatty acid synthase. Prog Lipid Res. 2003;42:289-317.

69. Leonard AE, Pereira SL, Sprecher H, Huang Y-S. Elongation of long-chain fatty acids. Prog Lipid Res. 2004;43:36-54.

70. Foster SP. Sex pheromone biosynthesis in the tortricid moth Planotortrix excessana (Walker) involves chain-shortening of palmitoleate and oleate. Arch Insect Biochem Physiol. 1998:37:158-67.

71. Hagström ÅK, Albre J, Tooman LK, Thirmawithana AH, Corcoran J, Löfstedt C, et al. A novel fatty acyl desaturase from the pheromone glands of Ctenopseustis obliquana and C. herana with specific z5-desaturase activity on myristic acid. J Chem Ecol. 2014;40:63-70.

72. Hunt MC, Alexson SEH. Novel functions of acyl-CoA thioesterases and acyltransferases as auxiliary enzymes in peroxisomal lipid metabolism. Prog Lipid Res. 2008;47:405-21.

73. Rosendal J, Ertbjerg P, Knudsen J. Characterization of ligand binding to acylCoA-binding protein. Biochem J. 1993;290(Pt 2):321-6.

74. Ohnishi A, Hull JJ, Matsumoto S. Targeted disruption of genes in the Bombyx mori sex pheromone biosynthetic pathway. Proc Natl Acad Sci U S A. 2006;103:4398-403.

75. Ohnishi A, Hashimoto K, Imai K, Matsumoto S. Functional characterization of the Bombyx mori fatty acid transport protein (BmFATP) within the silkmoth pheromone gland. J Biol Chem. 2009;284:5128-36.

76. Coleman RA, Lee DP. Enzymes of triacylglycerol synthesis and their regulation. Prog Lipid Res. 2004:43:134-76.

77. Shindou H, Hishikawa D, Harayama T, Yuki K, Shimizu T. Recent progress on acyl CoA: lysophospholipid acyltransferase research. J Lipid Res. 2009; 50(Supplement):S46-51.

78. Casida JE, Quistad GB. Organophosphate toxicology: safety aspects of nonacetylcholinesterase secondary targets. Chem Res Toxicol. 2004;17: 983-98.

79. Leal WS, Nikonova L, Peng G. Disulfide structure of the pheromone binding protein from the silkworm moth, Bombyx mori. FEBS Lett. 1999;464:85-90.

80. Benton R, Sachse S, Michnick SW, Vosshall LB. Atypical membrane topology and heteromeric function of Drosophila odorant receptors in vivo. PLOS Biol. 2006:4:e20.

81. Syed Z, Ishida Y, Taylor K, Kimbrell DA, Leal WS. Pheromone reception in fruit flies expressing a moth's odorant receptor. Proc Natl Acad Sci U S A. 2006;103:16538-43.

82. Tegoni M, Campanacci V, Cambillau C. Structural aspects of sexual attraction and chemical communication in insects. Trends Biochem Sci. 2004;29:257-64.

83. Vieira FG, Rozas J. Comparative genomics of the odorant-binding and chemosensory protein gene families across the Arthropoda: origin and evolutionary history of the chemosensory system. Genome Biol Evol. 2011:3:476-90.

84. Pelosi P, Zhou J-J, Ban LP, Calvello M. Soluble proteins in insect chemical communication. Cell Mol Life Sci CMLS. 2006;63:1658-76.

85. Angeli S, Ceron F, Scaloni A, Monti M, Monteforti G, Minnocci A, et al. Purification, structural characterization, cloning and immunocytochemical localization of chemoreception proteins from Schistocerca gregaria. Eur J Biochem. 1999:262:745-54

86. Zhang Y-N, Ye Z-F, Yang K, Dong S-L. Antenna-predominant and malebiased CSP19 of Sesamia inferens is able to bind the female sex pheromones and host plant volatiles. Gene. 2014;536:279-86.

87. Gu S-H, Wang S-Y, Zhang X-Y, Ji P, Liu J-T, Wang G-R, et al. Functional characterizations of chemosensory proteins of the alfalfa plant bug Adelphocoris lineolatus indicate their involvement in host recognition. PLoS One. 2012;7:e42871 
88. Vogt RG, Große-Wilde E, Zhou J-J. The Lepidoptera odorant binding protein gene family: gene gain and loss within the GOBP/PBP complex of moths and butterflies. Insect Biochem Mol Biol. 2015;62:142-53.

89. Newcomb RD, Sirey TM, Rassam M, Greenwood DR. Pheromone binding proteins of Epiphyas postvittana (Lepidoptera: Tortricidae) are encoded at a single locus. Insect Biochem Mol Biol. 2002;32:1543-54.

90. Callahan FE, Vogt RG, Tucker ML, Dickens JC, Mattoo AK. High level expression of "male specific" pheromone binding proteins (PBPS) in the antennae of female noctuiid moths. Insect Biochem Mol Biol. 2000;30:507-14.

91. Maida R, Ziegelberger G, Kaissling K-E. Ligand binding to six recombinant pheromone-binding proteins of Antheraea polyphemus and Antheraea pernyi. J Comp Physiol B. 2003;173:565-73.

92. Sirey TM. Pheromone binding proteins of New Zealand leafroller moths. Thesis. Auckland: University of Auckland; 2000

93. Langhoff P. Speciation genes in native New Zealand leafroller moths. Thesis. ResearchSpace@Auckland; 2010. https://researchspace.auckland.ac.nz/ handle/2292/5718. Accessed 18 Jan 2018.

94. Benton R, Vannice KS, Vosshall LB. An essential role for a CD36-related receptor in pheromone detection in Drosophila. Nature. 2007;450:289-93.

95. Jin X, Ha TS, Smith DP. SNMP is a signaling component required for pheromone sensitivity in Drosophila. Proc Natl Acad Sci. 2008;105:10996-1001.

96. Rogers ME, Krieger J, Vogt RG. Antennal SNMPs (sensory neuron membrane proteins) of Lepidoptera define a unique family of invertebrate CD36-like proteins. J Neurobiol. 2001;49:47-61.

97. Vogt RG, Miller NE, Litvack R, Fandino RA, Sparks J, Staples J, et al. The insect SNMP gene family. Insect Biochem Mol Biol. 2009;39:448-56.

98. Gu S-H, Wu K-M, Guo Y-Y, Pickett JA, Field LM, Zhou J-J, et al. Identification of genes expressed in the sex pheromone gland of the black cutworm Agrotis ipsilon with putative roles in sex pheromone biosynthesis and transport. BMC Genomics. 2013;14:636.

99. Benton R, Vannice KS, Gomez-Diaz C, Vosshall LB. Variant ionotropic glutamate receptors as chemosensory receptors in Drosophila. Cell. 2009;136:149-62.

100. Croset V, Rytz R, Cummins SF, Budd A, Brawand D, Kaessmann H, et al. Ancient protostome origin of chemosensory ionotropic glutamate receptors and the evolution of insect taste and olfaction. PLoS Genet. 2010;6: e1001064

101. Robertson HM, Warr CG, Carlson JR. Molecular evolution of the insect chemoreceptor gene superfamily in Drosophila melanogaster. Proc Natl Acad Sci U S A. 2003;100(Suppl 2):14537-42.

102. Abuin L, Bargeton B, Ulbrich MH, Isacoff EY, Kellenberger S, Benton R. Functional architecture of olfactory ionotropic glutamate receptors. Neuron. 2011;69:44-60

103. Rytz R, Croset V, Benton R. Ionotropic receptors (IRs): chemosensory ionotropic glutamate receptors in Drosophila and beyond. Insect Biochem Mol Biol. 2013;43:888-97.

104. Shanbhag SR, Singh K, Singh RN. Fine structure and primary sensory projections of sensilla located in the sacculus of the antenna of Drosophila melanogaster. Cell Tissue Res. 1995;282:237-49.

105. Vogt RG. Molecular basis of pheromone detection in insects. In: Gilbert LI, latrou K, Gill SS, editors. Comprehensive molecular insect science. Amsterdam: Elsevier; 2005. p. 753-803.

106. Durand N, Carot-Sans G, Chertemps T, Montagné N, Jacquin-Joly E, Debernard $\mathrm{S}$, et al. A diversity of putative carboxylesterases are expressed in the antennae of the noctuid moth Spodoptera littoralis. Insect Mol Biol. 2010;19:87-97.

107. Field LM, Devonshire AL. Evidence that the E4 and FE4 esterase genes responsible for insecticide resistance in the aphid Myzus persicae (Sulzer) are part of a gene family. Biochem J. 1998;330(Pt 1):169-73.

108. Bass C, Puinean AM, Zimmer CT, Denholm I, Field LM, Foster SP, et al. The evolution of insecticide resistance in the peach potato aphid, Myzus persicae. Insect Biochem Mol Biol. 2014;51:41-51.

109. Newcomb RD, Campbell PM, Ollis DL, Cheah E, Russell RJ, Oakeshott JG. A single amino acid substitution converts a carboxylesterase to an organophosphorus hydrolase and confers insecticide resistance on a blowfly. Proc Natl Acad Sci U S A. 1997;94:7464-8.

110. Wu W, Zhu J, Millar J, Löfstedt C. A comparative study of sex pheromone biosynthesis in two strains of the turnip moth, Agrotis segetum, producing different ratios of sex pheromone components. Insect Biochem Mol Biol. 1998;28:895-900.
111. Koutroumpa FA, Groot AT, Dekker T, Heckel DG. Genetic mapping of male pheromone response in the European corn borer identifies candidate genes regulating neurogenesis. Proc Natl Acad Sci U S A. 2016;113:E6401-8.

112. Hansson BS, Löfstedt C, Foster SP. Z-linked inheritance of male olfactory response to sex pheromone components in two species of tortricid moths, Ctenopseustis obliquana and Ctenopseustis sp. Entomol Exp Appl. 1989:53:137-45.

113. Clyne PJ, Certel SJ, de Bruyne M, Zaslavsky L, Johnson WA, Carlson JR. The odor specificities of a subset of olfactory receptor neurons are governed by Acj6, a POU-domain transcription factor. Neuron. 1999;22:339-47.

114. Tichy AL, Ray A, Carlson JR. A new Drosophila POU gene, pdm3, acts in odor receptor expression and axon targeting of olfactory neurons. J Neurosci. 2008;28:7121-9.

115. Brito NF, Moreira MF, Melo ACA. A look inside odorant-binding proteins in insect chemoreception. J Insect Physiol. 2016;95:51-65.

116. Jacquin-Joly E, Vogt RG, François M-C, Meillour PN-L. Functional and expression pattern analysis of chemosensory proteins expressed in antennae and pheromonal gland of Mamestra brassicae. Chem Senses. 2001;26:833-44.

117. Du G, Prestwich GD. Protein structure encodes the ligand binding specificity in pheromone binding proteins. Biochemistry (Mosc). 1995;34:8726-32.

118. Bette S, Breer H, Krieger J. Probing a pheromone binding protein of the silkmoth Antheraea polyphemus by endogenous tryptophan fluorescence. Insect Biochem Mol Biol. 2002;32:241-6

119. Große-Wilde E, Svatoš A, Krieger J. A pheromone-binding protein mediates the bombykol-induced activation of a pheromone receptor in vitro. Chem Senses. 2006:31:547-55.

120. Jordan MD, Stanley D, Marshall SDG, De Silva D, Crowhurst RN, Gleave AP, et al. Expressed sequence tags and proteomics of antennae from the tortricid moth, Epiphyas postvittana. Insect Mol Biol. 2008;17:361-73.

121. Wray GA. The evolutionary significance of cis-regulatory mutations. Nat Rev Genet. 2007:8:206-16.

122. Groot AT, Schöfl G, Inglis O, Donnerhacke S, Classen A, Schmalz A, et al. Within-population variability in a moth sex pheromone blend: genetic basis and behavioural consequences. Proc R Soc Lond B Biol Sci. 2014;281: 20133054

123. Duménil C, Judd GJR, Bosch D, Baldessari M, Gemeno C, Groot AT. Intraspecific variation in female sex pheromone of the codling moth Cydia pomonella. Insects. 2014;5:705-21.

\section{Submit your next manuscript to BioMed Central and we will help you at every step:}

- We accept pre-submission inquiries

- Our selector tool helps you to find the most relevant journal

- We provide round the clock customer support

- Convenient online submission

- Thorough peer review

- Inclusion in PubMed and all major indexing services

- Maximum visibility for your research

Submit your manuscript at www.biomedcentral.com/submit 